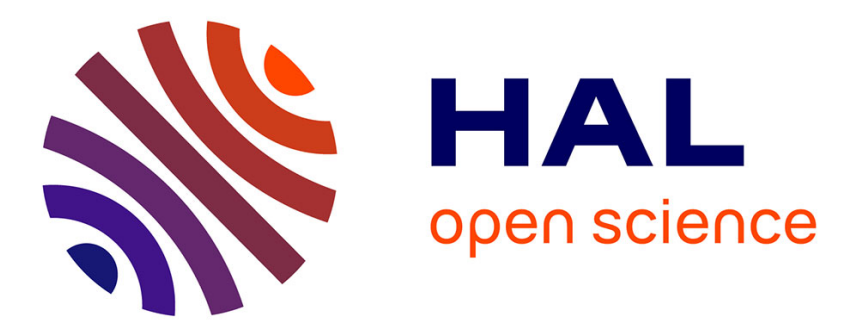

\title{
Revue Systématique de la Littérature sur le Soutien à la Sécurité des Opérations de Drones
}

Balita H Rakotonarivo, Stéphane Conversy, Nicolas Drougard, Jérémie Garcia

\section{To cite this version:}

Balita H Rakotonarivo, Stéphane Conversy, Nicolas Drougard, Jérémie Garcia. Revue Systématique de la Littérature sur le Soutien à la Sécurité des Opérations de Drones. 32e Conférence Francophone sur l'Interaction Homme-Machine (IHM '20.21), Apr 2021, Metz, France. 10.1145/3450522.3451328 . hal-03217297

\section{HAL Id: hal-03217297 \\ https://hal-enac.archives-ouvertes.fr/hal-03217297}

Submitted on 4 May 2021

HAL is a multi-disciplinary open access archive for the deposit and dissemination of scientific research documents, whether they are published or not. The documents may come from teaching and research institutions in France or abroad, or from public or private research centers.
L'archive ouverte pluridisciplinaire HAL, est destinée au dépôt et à la diffusion de documents scientifiques de niveau recherche, publiés ou non, émanant des établissements d'enseignement et de recherche français ou étrangers, des laboratoires publics ou privés. 


\section{Revue Systématique de la Littérature sur le Soutien à la Sécurité des Opérations de Drones}

Systematic Literature Review of Safety Support for Drones Operations

BALITA H. RAKOTONARIVO, ENAC - Université de Toulouse, France

NICOLAS DROUGARD, ISAE-SUPAERO, France

STÉPHANE CONVERSY, ENAC - Université de Toulouse, France

JÉRÉMIE GARCIA, ENAC - Université de Toulouse, France

CCS Concepts: • Human-centered computing $\rightarrow$ Interactive systems and tools; $\bullet$ General and reference $\rightarrow$ Surveys and overviews.

Additional Key Words and Phrases: UAS, mission, risk assessment, authorization, UTM, U-space, Human-machine interaction Dans cet article, nous nous intéressons au soutien à la sécurité des opérations de drones. Il s'agit d'un enjeu primordial pour les exploitants qui doivent se conformer au cadre légal. Nous présentons tout d'abord les systèmes de drones et la réglementation européenne. Ensuite nous décrivons une revue systématique de la littérature sur cette thématique. L'identification des approches principales en résulte : "Voir \& Éviter", Interactions Humain-Machine et Facteurs Humains (IHM/FH), intégrité de l'aéronef, analyses de sécurité, planification de trajectoires, géorepérage, cyber-sécurité et gestion du trafic de drones. Nous analysons plus en détail les contributions liées à l'IHM en identifiant les tâches utilisateurs, les recommandations pour la conception d'interactions améliorant la sécurité des opérations de drones et les perspectives de recherche. Enfin, nous discutons des aspects peu couverts dans les articles examinés.

Mots-clés additionnels : UAS, mission, analyse de risque, demande d'autorisation, UTM, U-space, Interaction Humain-Machine

\section{ACM Reference Format:}

Balita H. Rakotonarivo, Nicolas Drougard, Stéphane Conversy, and Jérémie Garcia. 2021. Revue Systématique de la Littérature sur le Soutien à la Sécurité des Opérations de Drones. In IHM '20'21:32e conférence Francophone sur l'Interaction Homme-Machine, April 13-16, 2021, Metz, France. ACM, New York, NY, USA, 24 pages. https://doi.org/10.1145/3450522.3451328

\section{INTRODUCTION}

Les drones sont de plus en plus utilisés pour des tâches de loisirs comme la photographie, mais également par des exploitants professionnels pour l'aide au sauvetage, la livraison de médicaments ou encore la surveillance de zones difficilement accessibles comme les voies de chemin de fer. Ainsi, de nouvelles applications apparaissent grâce à l'amélioration de l'autonomie et des capteurs embarqués des appareils [105].

Comme toute activité aéronautique, les opérations de drones présentent des risques qui peuvent avoir des conséquences dramatiques pour les biens et les personnes [117]. La réglementation de sécurité applicable aux drones a deux

Permission to make digital or hard copies of all or part of this work for personal or classroom use is granted without fee provided that copies are not made or distributed for profit or commercial advantage and that copies bear this notice and the full citation on the first page. Copyrights for components of this work owned by others than ACM must be honored. Abstracting with credit is permitted. To copy otherwise, or republish, to post on servers or to redistribute to lists, requires prior specific permission and/or a fee. Request permissions from permissions@acm.org.

(c) 2021 Association for Computing Machinery.

Manuscript submitted to ACM 
objectifs principaux. D’une part, elle assure la sécurité des autres usagers de l'espace aérien en réduisant le risque de collision en vol. D’autre part, elle assure la sécurité des biens et des personnes au sol en limitant le risque de crash. ${ }^{1}$

Depuis le premier janvier 2021, une réglementation européenne sur les Unmanned Aircraft System, ou UAS - autre dénomination des drones, est applicable dans l'ensemble des États membres de l'Union européenne, ainsi qu'en Islande, au Liechtenstein, en Norvège, et en Suisse. L'approche mise en oeuvre pour garantir la sécurité des missions de drones consiste à identifier les risques et à fournir des moyens de mitigation pour les réduire en dessous de seuils acceptables. Par exemple, le risque induit par la proximité d'une zone de vol en parachute pourra être mitigée par la réalisation de la mission considérée à une heure pour laquelle la probabilité de croiser un parachutiste est très faible; ou la demande d'une zone réservée temporairement au drone seul.

Des scénarios types, dont les risques sont déjà caractérisés, ont été décrits et permettent aux exploitants de préparer et réaliser des missions qui correspondent à ces scénarios en remplissant une déclaration. Certaines opérations qui ne correspondent à aucun de ces scénarios type, comme des missions dites de grande élongation pendant lesquelles le drone parcourt plusieurs kilomètres (ex : la surveillance de chemin de fer) nécessitent une autorisation des autorités pour pouvoir être réalisées. Dans ce cas, une boucle impliquant le client, l'exploitant de drones, le télépilote, le régulateur, les autorités de la gestion du trafic aérien et préfectorales se met en place. Les interactions humain-machine sont primordiales pour maintenir la sécurité des opérations planifiées.

Dans cet article, nous nous intéressons aux méthodes et outils existants qui permettent de soutenir la sécurité dans les opérations de drones, durant toutes les étapes de la mission : préparation, demande d'autorisation et réalisation. Au sein de la communauté IHM et robotique, plusieurs états de l'art recensent des travaux qui visent à faciliter les missions impliquant l'opération de drones. De nombreuses facettes sont couvertes comme la conception de drones [29], l'optimisation de trajectoires [1], les aspects de vie-privée ou de résistance aux attaques malveillantes [5], les interactions avec les drones pour du pilotage de loisir [51, 105], l'aide au contrôle d'essaims de drones [50], ou encore les outils et interactions disponibles pour les télépilotes pendant les phases de vol [87]. Cependant, aucun de ces états de l'art ne traite explicitement du support à la sécurité des missions de drones. A notre connaissance, l'interaction humain-machine pour la sécurité des opérations de drones ne semble que très peu étudiée. Ce manque constitue un frein au développement de cette activité en toute sécurité.

Cet article débute par la description du système de drones civils, de la réglementation européenne et du concept de U-Space caractérisant la gestion du trafic aérien qui est envisagée pour les drones. Nous insistons sur les aspects liés à la sécurité des opérations pertinents pour appréhender les travaux existants sur cette thématique. L'article continue avec une revue systématique de la littérature des approches existantes pour soutenir la sécurité des mission de drones, soit des méthodes permettant d'évaluer la sécurité ou des moyens utilisés pour mitiger les risques a priori ainsi que pendant la phase vol. Nous détaillons les travaux mettant en oeuvre des approches IHM et leurs contributions afin d'aider les concepteurs de futurs systèmes. Nous identifions également des usages ou éléments du système de drones qui sont peu couverts par la littérature. Enfin, une discussion permet de soulever des thématiques à explorer pour améliorer la sécurité des vols et le processus de préparation de mission de drones, pour pouvoir orienter de futurs travaux de recherche dans ce domaine.

${ }^{1}$ Notons que le terme sécurité concerne la préventions d'évènements involontaires pouvant se produire, alors que le terme sûreté concerne la prévention des actions malveillantes volontaires. 


\section{LES OPÉRATIONS DE DRONES}

La réalisation d'une mission avec un ou plusieurs drones nécessite la mise en oeuvre de plusieurs sous-systèmes permettant le contrôle, la commande, la communication ou le pilotage dans un cadre légal formel. En France, le guide des activités particulière [42] établit que "l'utilisation en extérieur d'engins volants, même lorsqu'ils sont de petite taille, qu'ils ne transportent personne à leur bord et qu'ils sont utilisés à basse hauteur, est considérée comme une activité aérienne et relève donc de la réglementation applicable à l'aviation civile." Dans cette partie, nous introduisons et nous décrivons les éléments nécessaires pour comprendre le contexte et les usages des drones. Nous mettrons en avant les points concernant la sécurité des opérations.

\subsection{Système de drones}

Un drone, aussi appelé Unmanned Aircraft System (UAS), désigne tout aéronef sans pilote à bord, pouvant être exploité de manière autonome ou être piloté à distance avec l'équipement servant à le contrôler [31,61].

Les systèmes de drones [23] sont en général composés de trois segments principaux : le segment air, soit le ou les aéronefs composés de divers équipements (propulsion, batterie, électronique embarquée et capteurs) ainsi que leur charge utile (une caméra, des poches de sang, ou même des passagers non-pilote); le segment sol, qui comprend la station de contrôle au sol, les éléments pour le décollage et la récupération; le segment de communication, permettant le lien entre le segment sol et le segment air, et pouvant inclure l'usage de satellites. Ces segments sont mis en oeuvre pour réaliser des missions de divers types comme expliqué dans ce qui suit.

\subsection{Missions de drones et domaines d'application}

Historiquement, les drones servaient aux missions "Dirty, Dull, Dangerous" (3D) pour "Sales, monotones et dangereuses". Aujourd'hui, il est difficile d'établir une liste exhaustive des domaines d'application actuels car les nouveaux usages se multiplient. Au delà d'une pratique de loisir, plusieurs grands domaines d'applications peuvent être dégagés : télémesures, inspections industrielles, photographie aérienne, surveillance et reconnaissance, réponse aux urgences, collecte d'informations atmosphériques ainsi que des applications requérant des interactions physiques avec des substances, des matériaux et des objets - dont la livraison par drones fait partie [18].

D'un point de vue technique, on distingue deux types de missions : les missions en vue directe, ou Visual Line of Sight (VLOS), dans lesquelles le pilote à distance est capable de maintenir un contact visuel continu avec le drone, et les missions hors-vue, ou Beyond Visual Line of Sight (BVLOS), lorsque le contact visuel ne peut être maintenu pour cause de distance, d'obstacles ou de climat [30]. Ces types de missions font partie des éléments pris en compte dans les règles décrites dans la section suivante.

\subsection{Réglementation européenne}

La Commission Européenne a publié en juin 2019 deux règlements portant sur les UAS qui expriment des exigences sur l'importation et la fabrication des drones, ainsi que sur les règles et procédures applicables à leur exploitation. En France, c'est la Direction Générale de l'Aviation Civile (DGAC) qui met en oeuvre cette réglementation [79].

La hauteur maximale à laquelle les drones pourront s'élever est fixée à $120 \mathrm{~m}$ dans l'espace aérien à très basse altitude ou Very Low Level (VLL). Toutefois, cet espace aérien n'est pas forcément vide et d'autres usagers peuvent aussi l'exploiter (planeur, parachutisme, hélicoptères). Les opérations sont classées en trois catégories d'exploitation : ouverte, spécifique ou certifiée, selon les risques qu'elles présentent. Les risques considérés concernent les autres objets présents 
dans les airs (risque air) ou ceux présents au sol (risque sol) [31]. Ces catégories sont décrites dans les sections suivantes dans un ordre de risques croissants ${ }^{2}$.

2.3.1 Catégorie d'exploitation ouverte. La catégorie ouverte permet les opérations des UAS à faible risque. Ils s'agit de vols en vue directe, avec des aéronefs de masse inférieure à $25 \mathrm{~kg}$. Dans cette catégories, les vols ne sont soumis à aucune autorisation préalable. Selon le poids du drone et sa vitesse, il est possible de survoler des personnes (masse inférieure à $250 \mathrm{~g}$ ) ou de voler à proximité (masse inférieure à $900 \mathrm{~g}$ ). Pour les drones plus lourds, une distance minimale avec les personnes, zones résidentielles, commerciales et industrielles doit être maintenue (au moins 30m pour un drone de moins de $4 \mathrm{~kg}$ et au moins $150 \mathrm{~m}$ sinon).

2.3.2 Catégorie d'exploitation spécifique. La catégorie spécifique permet des opérations à risques modérés, quand les conditions en catégorie ouverte ne sont plus respectées, comme lorsque le vol est hors-vue ou est réalisé dans des endroits représentant un risque plus important pour les tiers (en zone peuplée, à proximité d'un aérodrome, etc.). Pour voler dans cette catégorie, il est nécessaire de demander et d'obtenir une autorisation de la Direction de la Sécurité de l'Aviation Civile (DSAC) en France, qui est l'autorité nationale compétente chargée de la surveillance et de la certification dans le domaine de l'aviation civile.

Les autorisations sont délivrées sur la base d'une analyse de risque [30]. Un moyen acceptable de conformité pour cette analyse, reconnu par l'Agence Européenne de Sécurité Aérienne (AESA), est une méthodologie nommée SORA (Specific Operations Risk Assessment ou Analyse de sécurité pour les opérations de la catégorie spécifique). A partir de l'analyse du risque air et du risque sol, cette méthodologie permet d'établir des objectifs de sécurité opérationnels à atteindre, afin de déterminer les moyens à mettre en œuvre pour rendre ces risques acceptables. La réglementation européenne prévoit aussi que l'on puisse voler avec une simple déclaration dans le cadre de scénarios standards (STS). Si l'opération de drones se conforme à ces exigences, alors une simple déclaration est suffisante car les risques ont déjà été évalués de façon générique pour ces scénarios.

2.3.3 Catégorie d'exploitation certifiée. Cette catégorie concerne les drones d'une dimension caractéristique supérieure ou égale à $3 \mathrm{~m}$, ou conçus pour être exploités au-dessus d'un rassemblement de personnes, pour le transport de personnes, ou pour le transport de marchandises dangereuses, exigeant donc une grande robustesse afin d'atténuer les risques pour les tiers en cas d'accident. Ces vols nécessitent la certification du drone ainsi que la certification de l'exploitant et, le cas échéant, l'octroi d'une licence au pilote à distance dans un cadre similaire à celui de l'aviation générale.

\subsection{UTM et U-Space}

Le nombre de drones en exploitation va augmenter considérablement dans les prochaines années. Ainsi, même si la hauteur maximale de 120 mètres au-dessus du sol offre une garantie de séparation par rapport à l'aviation traditionnelle, cet espace aérien ne sera plus aussi vide et de nombreux problèmes d'accès concurrents devront y être résolus. Actuellement, l'UAS Traffic Management (UTM) [65] permet aux drones de voler dans l'espace aérien de basse altitude. La Commission Européenne souhaite créer un système de gestion du trafic des drones appelé U-Space.

U-Space est un ensemble de services pour garantir une insertion sûre, sécurisée et efficace des drones dans l'espace aérien [101]. Il est prévu une mise en oeuvre de ce système selon 4 étapes incrémentales (U1 à U4) sous formes de groupes de services pour le U-Space [100]. U1 (fondation) : couvrant l'enregistrement en ligne, l'identification et le géorepérage.

${ }^{2}$ le détail des catégories est disponible sous forme d'infographies sur cette page http://drone-chair.enac.fr/rpas-chair/3-infographies-pour-y-voir-plusclair-reglementation-europeenne-des-drones/ 
U2 (initiale) : couvrant la gestion des opérations de drones comprenant la planification des vols, l'autorisation des missions, le tracking et l'interface avec le trafic aérien conventionnel. U3 (avancée) : couvrant le support aux opérations complexes dans les zones denses comprenant la détection de conflits ou les fonctionnalités d'évitement de collision. U4 (totale) : un très haut niveau d'automatisation, de connectivité et de numérisation liés avec l'aviation générale.

\subsection{Bilan}

Pour conclure cette section, l'utilisation des drones, en dehors des activités de loisirs, est une activité complexe, fortement réglementée, avec de multiples acteurs (opérateurs, pilotes, autorités..) et des interactions nombreuses et diverses entre ceux-ci. La réglementation européenne applicable depuis janvier 2021 met en avant de nombreuses interactions entre les acteurs avec un haut niveau de digitalisation et de connectivité dans les services envisagés. Les aspects de sécurité, de sûreté, de respect de la vie privée et de l'environnement sont primordiaux, et des catégories d'opérations sont définies selon les risques qu'elles présentent. Dans les cas nominaux, les procédures pour permettre l'opération sont relativement simples via des déclarations sous forme de formulaires en ligne. Pour des cas non nominaux, la demande d'autorisation ainsi que la production d'études de sécurité deviennent nécessaires. Dans cet article, nous nous focalisons sur le support à la sécurité dans les opérations de drones. Cet aspect va prendre de plus en plus d'importance avec l'augmentation des missions de drones et la mise en oeuvre de services U-Space. Afin de pouvoir étudier et caractériser l'existant, une revue systématique de la littérature a été menée sur cette thématique.

\section{MÉTHOdOLOGIE}

Cette section décrit le processus utilisé pour réaliser une revue systématique de la littérature (RSL) et sur notre méthodologie d'analyse des résultats. Même si une approche rigoureuse n'exclut pas nos connaissance préalables et des potentiels biais, notre objectif a été de mener un travail systématique à toutes les étapes afin que les lecteurs puissent inspecter, évaluer et exploiter nos résultats [108].

\subsection{Questions de recherche}

Notre objectif est l'amélioration des Interactions Humain-Machine (IHM) afin de maximiser la sécurité lors de la préparation, la demande d'autorisation et la réalisation des missions de drones. Nos questions de recherche sont :

- RQ1 : Quelles sont les types de missions qui bénéficient de soutien à la sécurité et celles qui sont peu représentées?

- RQ2 : Quelles sont les approches existantes du soutien à la sécurité des missions de drones?

- RQ3 : Quelle est la proportion d'approches s'appuyant sur l'IHM?

- RQ4 : Quelles sont les contributions du domaine de l'IHM dans les articles scientifiques traitant du soutien à la sécurité des missions de drones?

\subsection{Réalisation de la RSL}

Les bases de données utilisées sont Web Of Science (WoS) et la Digital Library de l'Association for Computing Machinery (DL ACM). DL ACM contient les références de 6 des 10 publications en Interactions Homme Machine ayant un h5-index le plus élevé [99]. WoS est retenu car faisant partie des ressources électroniques accessibles par les auteurs. Elles permettent de rassembler les publications de nombreuses collections scientifiques notamment IEEE Xplore.

Les mots clés utilisés sont "safety" et "unmanned". En effet, la terminologie employée confond parfois UAS (Unmanned Aircraft System), c.-à-d. le système de drones, UAS (Unmanned Autonomous System) c.-à-d. le système de drones 


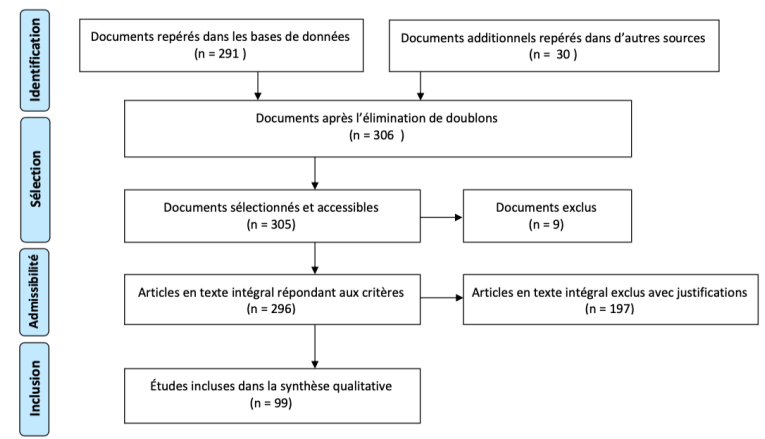

Fig. 1. PRISMA Flowchart de la RSL "Safety for Unmanned"

autonomes, et UAV (Unmanned Aerial Vehicle) c.-à-d. l'aéronef; "unmanned" pourra donc couvrir ces cas de figure. De même, "unmanned" pourra aussi être remplacé par RPAS (Remotely Piloted Aircraft System) et drone. La recherche s'est faite parmi les titres des articles, leurs résumés et leurs mots-clés. Nos essais préalables ont montré que la recherche avancée sur Google Scholar n'était pas assez spécifique, raison pour laquelle cette base n'a pas été retenue ici. De plus, des articles issus d'une première itération de la RSL, avec les mots clés "safety", "human machine interaction", "drone" et "mission" sont ajoutés, la méthode permettant d'inclure des données pertinentes qui ne font pas nécessairement partie des bases de données sélectionnées [41].

La figure 1 montre le déroulement et le résultat de la sélection des articles retenus, selon la présentation standard préconisée par le Preferred Reporting Items for Systematic Reviews and Meta-Analyses (PRISMA) [80]. L'extraction a été menée par une seule personne mais avec une vérification par un tiers, afin de minimiser les biais d'évaluation.

Des 321 articles originaux, 160 proviennent de WoS et 131 de la DL ACM et en tout, 306 sont uniques. Nous avons ensuite utilisé les critères d'exclusion suivant : langues autre que l'anglais ou le français; ne traite pas d'aéronautique; types autres que chapitre de livre ou article; publié avant 2014; texte intégral inaccessible; pas de lien avec la sécurité des missions de drones; application pour la sécurité en utilisant des drones. Ce filtrage a été effectué par une seule personne avec des vérifications aléatoires par une autre personne. Un article n'était pas accessible et 9 autres n'étaient pas des articles. Parmi les 296 restants, 197 ne traitaient pas du soutien à la sécurité pour les missions de drones; ainsi il en reste 99 à analyser.

\subsection{Analyse}

Afin de permettre une analyse systématique et exhaustive de chaque article, nous avons utilisé une variante de la méthode de questionnement [8]. Elle peut servir de garant pour le principe de reproductibilité [20] et s'adapte à différentes problématiques. Nous proposons ainsi la déclinaison des questions Qui, Quoi, Où, Quand, Combien, Comment et Pourquoi ou QQOQCCP pour analyser les articles relatifs à la sécurité pour le soutien aux missions de drones. Les déclinaisons sont données sur les tables ?? et ??. La question "Pourquoi ?" n'est pas prise en compte car les articles retenus concernent tous le soutien à la sécurité des missions de drones.

Afin de répondre à RQ2 et RQ3, nous avons attribué une catégorie principale à chaque article pour la question “Comment?”, c'est-à-dire quelle est l'approche principale présentée dans chaque article. Les catégories finales ont été raffinées itérativement par deux personnes. Pour répondre à RQ4, nous avons aussi considéré les articles avec une 


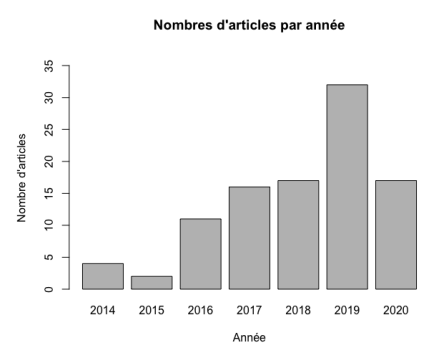

Fig. 2. Nombre d'articles par année depuis 2014

composante IHM même si ce n'était pas la composante principale. Pour chacun de ces articles, nous avons attribué une catégorie d'approche issue de l'IHM telles que Interface Graphique, Facteurs Humains ou encore Technique d'Interaction.

\subsection{Résultats}

Dans cette section, nous présentons un ensemble de perspectives concernant les recherches actuelles sur le soutien à la sécurité des missions de drones. Les résultats sont issus de l'analyse des articles retenus par notre RSL ( $\mathrm{n}=99)$. Ces résultats offrent des conclusions approfondies sur les cas d'études considérés (type de drones, phase de vol...) ainsi que sur les approches de recherches, notamment en IHM. La table ?? présente les résultats relatifs à la grille d'analyse fournie par la méthode QQOQCCP.

Pour certaines questions comme “Qui ?” ou “Quoi?”, certains articles couvrent plusieurs catégories. Lorsque c'est le cas, nous présentons les données sous plusieurs formes. Pour chaque réponse aux questions, nous donnons le nombre d'articles associés et le pourcentage sur l'ensemble des articles. Si les articles ont été associés à plusieurs réponses, nous traitons les données comme s'il s'agissait de questions à choix multiples et proposons un pourcentage sur le nombre d'articles mais également par rapport au nombre total de réponses. Pour les questions à choix multiples, nous utilisons le pourcentage par rapport au nombre total de réponses qui nous semble plus pertinent pour appréhender les proportions. La figure 2 présente le nombre d'articles par année. Depuis 2015, la tendance est à l'augmentation. L'extraction des données ayant été effectuée en juin 2020, cela ne permet pas d'observer ce phénomène pour 2020.

\subsection{Cas d'études considérés (RQ1)}

Qui ? Les bénéficiaires d'aide à la sécurité sont principalement les télépilote (46.83\%). Les prestataires de service de la navigation aérienne, incluant le contrôle aérien tel qu'il est connu actuellement et les futurs fournisseurs de service de l'UTM constituent $20.63 \%$ des articles, les exploitants $18.25 \%$ et les concepteurs-fabricants de drones $9.52 \%$. Enfin, les régulateurs constituent $4.76 \%$ des articles.

Quoi? Les drones de moins de $25 \mathrm{~kg}$ constituent $62.14 \%$ des articles, tandis que les plus lourds constituent $22.33 \%$ et le type de drones n'est pas mentionné pour 15.53\%. Les aéronefs autonomes, pour lesquels le pilote n'a aucune intervention à effectuer, constituent $39.39 \%$ des articles; ceux où le pilote reste nécessaire en constituent $8.59 \%$ même si le drone est hautement automatisé et cela n'a pas pu être déterminé pour deux articles, soit $2.02 \%$ Les voilures tournantes, ce qui inclut les quadricoptères, constituent $50.48 \%$ des articles alors que les voilures fixes en constituent $23.81 \%$ et que $25.71 \%$ des articles ne mentionnent pas le genre de drones considérés. Le segment air constitue $51.35 \%$ des articles, le segment sol constitue $37.84 \%$ des articles et le segment des communications $10.81 \%$. 
TABLE 1. Répartition des approches principales pour l'aide à la sécurité des opérations de drones.

\begin{tabular}{lccl}
\hline Approche principale & $\begin{array}{c}\text { Nombre } \\
\text { d'articles }\end{array}$ & Pourcentage & Références \\
\hline Intégrité de l'aéronef & 16 & $16,16 \%$ & {$[17,21,22,26,27,52,57,86,89,90,96,110-112,116,122]$} \\
\hline Cyber-sécurité & 7 & $7,07 \%$ & {$[6,28,58,93,107,124,124]$} \\
\hline Détecter et éviter & 20 & $20,20 \%$ & {$[7,13,32,35,43,44,46,56,60,73,74,77,82,84,91,92,109,118,123,125]$} \\
\hline Géorepérage & 3 & $3,03 \%$ & {$[48,59,104]$} \\
\hline $\begin{array}{l}\text { Interaction Homme-Machine } \\
\text { et Facteurs Humains }\end{array}$ & 17 & $17,17 \%$ & {$[2,9,12,19,24,47,64,67,68,71,72,83,85,94,95,106,119]$} \\
\hline Planification de trajectoires & 12 & $12,12 \%$ & {$[10,14,34,40,49,55,62,63,70,75,98,121,121]$} \\
\hline Analyse de sécurité & 14 & $14,14 \%$ & {$[3,4,25,36-38,38,39,54,66,69,81,88,102,113]$} \\
\hline Gestion du trafic de drones & 10 & $10,10 \%$ & {$[11,15,16,33,53,76,97,114,115,120]$} \\
\hline
\end{tabular}

Où? Si l'espace aérien non-contrôlé au-dessus du Very Low Level (VLL) constitue 1.96\% des articles, l'espace à très basse altitude en constitue 57.84\%. 17.65\% des articles sont concernés par l'espace aérien contrôlé, qui inclut les aérodromes alors que $22.55 \%$ ne mentionnent pas les caractéristiques de l'espace aérien où leurs opérations ont lieu. Pour ce qui est des caractéristiques de l'environnement survolé, les environnements urbains constituent $20.20 \%$ des articles, $58.59 \%$ ne les concernent pas, tandis que $21.21 \%$ ne le mentionnent pas. En effet, si les principales caractéristiques (contrôlé, non-contrôlé, VLL) ne sont pas indiquées, souvent le fait que l'environnement soit urbain ou pas n'est pas non plus communiqué. Les missions en BVLOS constituent $71.96 \%$ des articles, celles en VLOS $11.21 \%$ et $16.82 \%$ ne mentionnent pas le type de mission. L'applicabilité aux missions à longue élongation est possible pour $81,82 \%$ des articles mais $11,11 \%$ ne le mentionnent pas. L'intégration des drones et du trafic aérien avec pilotes à bords constitue $23.23 \%$ des articles par le biais de tests, simulation ou démonstration et $70.71 \%$ ne s'en préoccupent pas. Six articles ne le mentionnent pas $(6.06 \%)$.

Quand? La réalisation de la mission constitue $70.37 \%$ des articles. La préparation de celle-ci constitue $19.44 \%$. Un article concerne l'après réalisation de la mission (0.93\%), deux articles la réglementation (1.85\%), trois articles la demande d'autorisation (2.78\%) et cinq articles la fabrication des drones $(19.44 \%)$.

Combien? Les vols en solo constituent $56.57 \%$ des articles, les essaims de drones $13.13 \%$ et plusieurs drones partageant le même espace en constituent $31.31 \%$.

Le type de drones qui bénéficie le plus du soutien à la sécurité est celui des petits drones, avec $62,14 \%$ des réponses. Le moins représenté est celui des gros drones. Le type d'exploitation est en hors-vue ou BVLOS, avec 71,96\% des réponses. Les contributions, détaillées à la sous-section suivante, sont applicables aux opérations à grande élongation (OGE) pour $81,82 \%$ des réponses.

\subsection{Approches principales (RQ2 - RQ3)}

Huit approches principales ont émergé de l'analyse des articles, synthétisées dans le tableau 1.

La préservation de l'intégrité de l'aéronef constitue $16.16 \%$ des articles. Les thèmes sont principalement les systèmes embarqués dont les systèmes de commandes en vol [27], notamment la détection de défaillances $[17,21,22,57,89,90,96]$, la gestion des contingences [110], la conception de systèmes résilients [111, 122] et les aides pour l'atterrissage $[26,52,86,98,112,116]$.

La cyber-sécurité constitue $7.07 \%$ des réponses. L'enjeu est de protéger le système contre les attaques. Il s'agit de revues de la littérature $[6,58,124]$, du cryptage des communications $[28,103,107]$ et d'une proposition d'architecture de systèmes sécurisés [93]. 
TABLE 2. Répartition des catégories IHM/FH principales

\begin{tabular}{lccl}
\hline Catégories & Nombre d'articles & Pourcentage & Références \\
\hline facteurs humains & 5 & $18,52 \%$ & {$[9,12,64,72,94]$} \\
\hline interface graphique & 11 & $40,74 \%$ & {$[2,3,15,16,38,39,47,71,76,95,115]$} \\
\hline technique d'interaction & 2 & $7,41 \%$ & {$[85,106]$} \\
\hline visualisation d'information & 9 & $33,33 \%$ & {$[19,24,32,67,68,73,83,88,119]$} \\
\hline
\end{tabular}

"Détecter et Éviter" représente $20.20 \%$ des articles. Cette approche comprend la détection d'obstacles ou d'intrus dans une certaine zone de sécurité autour de l'aéronef en vol et selon le système, l'évitement automatique de collisions si nécessaire en plus de l'alerte. Les articles consistent en des revues de littérature où le sujet est traité [82, 92, 109], des améliorations d'algorithmes [7, 13, 32, 35, 43, 44, 56, 60, 74, 77, 84, 91, 118, 123, 125] ainsi que des systèmes (matériel et logiciel) fournissant la fonctionnalité de Détecter et Éviter [46, 73].

Le géorepérage constitue 3.03\% des réponses. La réglementation [30] la définit comme une fonction qui, sur la base des données fournies par le régulateur, détecte une violation potentielle des limites de l'espace aérien et en alerte les télépilotes, afin que ces derniers puissent agir de manière immédiate et efficace pour éviter cette violation. Des zones géographiques dans lesquelles l'aéronef doit évoluer (keep-in) ou qu'il doit éviter (keep-out) sont définies. Plusieurs algorithmes sont proposés par [48, 104] tandis que [59] propose la mise en oeuvre d'une zone d'interdiction de vol.

La catégorie des articles dont l'approche principale se base sur les interactions hommes-machines (IHM) et les facteurs humains $(\mathrm{FH})$ correspond à $17.17 \%$ du total des articles, soit 17 articles sur 99 . Une revue de littérature sur les facteurs humains relatifs aux opérations de drones est proposée [9]. Des travaux définissent des métriques pour la mesure des performances des télépilotes [94] ou évaluent la charge mentale de travail pour les contrôleurs gérant du trafic impliquant des drones [12]. L'amélioration des aides au pilotage et à la préparation de missions est aussi étudiée pour mieux identifier les pistes d'atterrissage par mauvaise visibilité [83], changer le point de vue du pilote pour faciliter les opérations dans les environnements complexes en 3D [106], utiliser plusieurs drones lors de mission de sauvetage [2], planifier des trajectoires en réalité virtuelle [85] ou communiquer, vérifier et valider les objectifs de mission de drones autonomes [95]. Les interfaces adaptatives pour le pilotage sont l'objet des travaux de [19, 64, 71, 72]. Pour la surveillance, on trouve des outils pour l'identification et le suivi des aéronefs $[67,68]$ ou des images-radar pour les contrôleurs afin d'intégrer les drones au trafic aérien [47] ou en milieu urbain [24].

La planification de mouvements, ou dans notre cas la planification de trajectoires, représente $12.12 \%$ des approches. La plupart des travaux proposent ou améliorent des algorithmes de calcul de trajectoires avant la mission [49, 55, 63, $75,98,121,121]$ ou de replanification en ligne $[14,34,40,70]$. Un article prend également en compte des paramètres opérationnels de la mission et propose des comparaisons par simulation [10].

Les méthodes d'analyses de sécurité représentent $14.14 \%$ des approches. Il s'agit de moyens pour évaluer les risques avant la construction des drones [69] et des systèmes [102], ou avant les opérations [4] avec des cartes de risques au sol en cas d'impact $[36,66,88]$ ou encore des méthodes formelles pour prédire la résilience des systèmes [81]. Ces méthodes concernent aussi l'exécution des opérations avec un système de recommandation utilisant les méthodes formelles [54]. Des outils pour générer automatiquement des dossiers de sécurité sont proposés [37-39] ainsi que des moyens d'identifier les changements entre plusieurs versions [3]. La méthode SORA est appliquée à un contexte de production multimédia multidrone [25]. Vierhauser et al. [113] proposent une méthodologie comprenant la définition de dossiers de sécurité pour les espaces aériens d'une part et des exigences et des contraintes pour les drones demandant 
à y évoluer d'autre part. Des moyens de vérification et validation entre les deux jeux de contraintes sont mis en oeuvre avant la mission puis pendant sa réalisation.

La catégorie "Gestion du trafic de drones", représente $10.10 \%$ des réponses. On trouve une revue de la littérature des régulations et technologies pour le vol urbain [120] ainsi que des suggestions pour les futurs systèmes et services $[11,53,76]$. Plusieurs travaux concernent des outils et concepts pour la planification de missions et le monitoring $[15,16,33,97,115]$. Certains permettent de simuler la gestion du trafic aérien pour tester et expérimenter [15, 114, 115].

\section{ANALYSE DES CONTRIBUTIONS DE L'IHM (RQ4)}

Afin de répondre à RQ4, nous avons centré notre analyse sur les dix-sept articles dont l'approche principale est IHM ou $\mathrm{FH}$, traités précédemment, ainsi que sur dix autres articles qui mentionnaient ou décrivaient des contributions IHM $[3,15,16,32,38,39,73,76,88,115]$. Pour chacun de ces vingt-sept articles, nous avons identifié une catégorie principale ainsi que les tâches des utilisateurs. La mention de l'utilisation de représentations géographiques a été déterminée. Nous avons également étudié les recommandations proposées pour la conception de systèmes et de missions de drone sûrs, ainsi que les perspectives de recherche suggérées.

\subsection{Catégories IHM}

La table 2 présente le nombre d'articles pour chacune des catégories identifiées. Les catégories "interfaces graphiques" (40,74\%) et "visualisation d'information" (33,33\%) sont les deux catégories les plus représentées. La différence entre les deux catégories tient principalement à la possibilité pour les utilisateurs d'agir sur le système pour modifier l'étude de sécurité ou le plan de vol par exemple. Il s'agit majoritairement d'outils permettant de créer une mission en plaçant des points sur une carte, et de visualisations de type image radar pour surveiller le bon déroulement de la mission. Pour les interfaces graphiques, on trouve également des éditeurs servant à l'analyse de sécurité. Pour la visualisation d'information, il y a aussi des instruments de vol comme des systèmes d'évitement de collision en vol entre aéronefs. La catégorie facteurs humains $(18,52 \%)$ comprend plusieurs travaux visant à la mesure de performance des pilotes ou des contrôleurs afin d'adapter les interfaces graphiques à leur charge mentale. Certaines approches utilisent des modèles de charge mentale en fonction des phases de vol de la mission pour proposer des adaptations. Enfin, la catégorie technique d'interaction (7,41\%) comprend deux articles traitant de techniques de réalité virtuelle pour le pilotage et la préparation de mission.

\subsection{Tâches utilisateur}

Nous avons identifié cinq tâches soutenues par des approches IHM ou FH. La répartition de ces tâches pour les 27 articles est présentée dans la table 3. La surveillance (42,50\%) consiste à s'assurer que la mission se déroule dans des conditions nominales. Plusieurs travaux proposent des visualisations sous forme d'image radar ou d'instruments de vols alors que d'autres ajoutent des représentations dédiées à la sécurité des vols comme les alertes en cas d'intrusion dans une zone interdite. Le pilotage (17,50\%) est facilité par des techniques d'interaction ou par des interfaces graphiques qui permettent aux utilisateurs de saisir des valeurs de vitesse ou des points géographiques à atteindre sur une carte. Les approches d'adaptation dynamique à partir de mesures de l'état cognitif du pilote ou du déroulement de la mission visent également à rendre le pilotage plus performant. La préparation de mission (15\%) consiste à définir les points d'intérêts sur une carte ou à planifier les déplacements du ou des drones avec des algorithmes pour assurer des trajectoires optimales. Le contrôle $(12,5 \%)$ consiste à aider les utilisateurs à assurer une séparation suffisante entre les drones et/ou 
TABLE 3. Répartition des tâches utilisateurs (40 réponses au total).

\begin{tabular}{lclcc}
\hline Tâches & $\begin{array}{c}\text { Nombre de } \\
\text { réponses }\end{array}$ & Références & $\begin{array}{c}\text { Pourcentage réponses } \\
\text { sur nombre articles }\end{array}$ & $\begin{array}{c}\text { Pourcentage réponses } \\
\text { sur nombre réponses }\end{array}$ \\
\hline Analyse de sécurité & 4 & {$[3,38,39,88]$} & $14,81 \%$ & $10,00 \%$ \\
\hline Contrôle & 5 & {$[9,12,47,64,76]$} & $18,52 \%$ & $12,50 \%$ \\
\hline Pilotage & 7 & {$[2,67,68,71,83,94,106]$} & $25,93 \%$ & $17,50 \%$ \\
\hline Préparation de mission & 6 & {$[15,16,85,88,95,115]$} & $22,22 \%$ & $15,00 \%$ \\
\hline Surveillance & 17 & {$[2,9,12,15,19,24,32,64,67,68$,} & $62,96 \%$ & $42,50 \%$ \\
\hline Demande d'autorisation & 1 & {$[1-73,76,95,115,119]$} & & $2,50 \%$ \\
\hline
\end{tabular}

TABLE 4. Répartition des représentations géographiques par tâches

\begin{tabular}{lclcccc}
\hline Tâches & $\begin{array}{c}\text { AVEC } \\
\text { représentation } \\
\text { géographique }\end{array}$ & Références & $\begin{array}{c}\text { SANS } \\
\text { représentation } \\
\text { géographique }\end{array}$ & Références & $\begin{array}{c}\text { Non- } \\
\text { mentionné }\end{array}$ & Références \\
\hline Analyse de sécurité & $1(25,00 \%)$ & {$[88]$} & $3(75,00 \%)$ & {$[3,38,39]$} & 0 & \\
\hline Contrôle & $2(40,00 \%)$ & {$[47,76]$} & 0 & $3(60,00 \%)$ & {$[9,12,64]$} \\
\hline Pilotage & $6(85,71 \%)$ & {$[2,67,68,83,94,106]$} & 0 & $1(14,29 \%)$ & {$[71]$} \\
\hline Préparation de mission & $6(100,00 \%)$ & {$[15,16,85,88,95,115]$} & 0 & 0 & \\
\hline Surveillance & $13(76,47 \%)$ & {$[2,15,19,24,32,67,68$,} & 0 & $4(23,53 \%)$ & {$[9,12,64,71]$} \\
\hline Demande d'autorisation & $1(100,00 \%)$ & {$[2,73,76,95,115,119]$} & & 0 & 0 & \\
\hline
\end{tabular}

les avions en gérant leurs altitudes, trajectoires et vitesses. Enfin, l'analyse de sécurité (10\%) consiste à exprimer, souvent de façon formelle, la mission avant son déroulement pour identifier les risques et les contre-mesures nécessaires.

\subsection{Représentations géographiques en fonction des tâches}

Nous avons analysé la présence de représentations géographiques, c'est-à-dire une représentation spatiale liée au contexte de la mission. Les résultats de cette répartition en fonction des tâches est donnée dans la table 4. Les tâches de "pilotage", "préparation de mission", "surveillance" et "demande d'autorisation" sont principalement basées sur des représentations géographiques (> 76,47\%). On observe certains cas non précisés mais aucun cas de représentation non basée sur la géographie pour ces tâches. Pour la tâche de contrôle, certains articles ne précisent pas les représentations envisagées (60\%) mais cette activité est fréquemment basée sur une image radar et aucun article ne décrit de représentation non basée sur la géographie. Pour la tâche "analyse de sécurité", un seul article [88] présente une représentation géographique, avec une visualisation des risques au sol affichée sur une carte. Les trois autres articles sont basés sur d'autres représentations comme des diagrammes [39], des graphes [38] ou des arbres [3].

\subsection{Recommandations pour la conception}

Nous avons analysé les recommandations formulées par les différents articles en lien avec l'IHM ou FH. Nous présentons dans cette section ces résultats selon plusieurs dimensions. Ces recommandations peuvent être mises en oeuvre par des concepteurs de systèmes futurs.

4.4.1 Adapter et/ou personnaliser les interfaces. Plusieurs articles donnent des suggestions sur la présentation des informations aux opérateurs lors des missions [2, 19, 39]. La définition d'une mission de drones inclut le plan de vol mais aussi le plan d'utilisation de la charge utile. Besada et al. indiquent que l'interface doit être adaptée à la mission et ainsi prendre en compte l'utilisation de la charge utile [15]. 
Selon Bonache-Seco et al. [19], les utilisateurs doivent pouvoir choisir et accéder rapidement aux informations et interactions qui leurs semblent pertinentes. En fonction du rôle des utilisateurs (régulateur, opérateur, pilote, contrôleur de la navigation), une visualisation différente et spécifique du système ainsi que de son état est préférable [39]. Pour Agrawal et al. [2], lors d'une mission de sauvetage, les télépilotes souhaitent mettre en plein écran automatiquement le retour visuel de la caméra dès qu'une victime est détectée, alors qu'un contrôleur souhaite disposer d'une vue d'ensemble des différents drones participants à cette mission et que le régulateur pourrait s'intéresser à un diagramme Bow-Tie des dangers identifiés [39].

Certains travaux recommandent d'adapter automatiquement les interfaces utilisateurs et leurs comportements en fonction de critères ergonomiques [19], ou physiologiques [64, 71]. Ainsi, le niveau de transparence de certains éléments peut dépendre du nombre de drones à surveiller [19]. Lim et al. [71] suggèrent de réaliser l'adaptation en fonction du stress et de la charge de travail mentale de l'utilisateur. Ces derniers peuvent être estimés directement par l'opérateur, ou calculés à partir des caractéristiques des tâches à accomplir ou encore provenir de mesures psycho-physiologiques. Pour Kistan et al. [64] l'adaptation dynamique des interfaces utilisateurs par des techniques d'apprentissage automatique selon l'état cognitif estimé de l'utilisateur permettrait d'augmenter la capacité et la sécurité du système.

4.4.2 Informer les utilisateurs sur l'environnement extérieur et le système de drone. Communiquer l'état de l'environnement extérieur est important selon Agrawal et al. [2]. Les auteurs suggèrent de fournir des indices visuels reconnaissables quand des conditions opérationnelles pouvant impacter le comportement du système sont rencontrées, par exemple en utilisant des pictogrammes lors des changements de conditions météorologiques. Pour aider les pilotes à pallier le manque d'informations sensorielles perçues par rapport à une présence physique, l'utilisation de plusieurs modalités (visuelle, haptique, sonore, textuelle) est recommandée par plusieurs articles [9, 71, 78]. Concernant le comportement du système dans son ensemble, l'interface doit pouvoir montrer la trajectoire récente du drone ainsi que sa trajectoire prévue [2]. Il est aussi suggéré d'expliquer en temps réel l'impact des changements des conditions opérationnelles sur le comportement du système, ou ce que le système est autorisé à faire [2]. Par exemple : "Vous rentrez dans une zone où la hauteur est limitée à $75 \mathrm{~m}$." ou "La visibilité actuelle est mauvaise. Les drones devront voler à vitesse réduite et plus près du pilote de sécurité ". Agrawal et al. recommandent également de présenter de façons discernable les différentes options possibles lorsqu'une action des utilisateurs est requise [2].

4.4.3 Éviter la surcharge d'information. Agrawal et al. [2] suggèrent de limiter l'affichage aux informations essentielles et de rendre les informations complémentaires disponibles sur demande. Ils recommandent également d'éviter d'afficher certaines grandeurs difficiles à interpréter en temps réel comme les coordonnées géographiques (latitude et longitude) contrairement aux valeurs de vitesse et d'altitude par rapport au sol. Ils suggèrent aussi de séparer les rôles d'opérateur de la charge utile et de télépilote, par exemple pour éviter une focalisation trop importante sur la localisation de la victime au détriment de la fonction de pilotage, durant une mission de recherche et sauvetage. Dans le cas d'une visualisation du trafic, Campaña et al. [24] conseille de pouvoir identifier rapidement les zones où une intervention est requise via des alertes sans créer une surcharge d'information ni augmenter la charge de travail.

4.4.4 Alerter efficacement en fonction du contexte. Certains articles suggèrent que les caractéristiques des alarmes doivent dépendre de la proximité du danger ou de sa probabilité $[15,71]$. Un exemple donné est celui du système d'alerte de trafic et d'évitement de collision (TCAS), dont l'interface et le son varient en fonction de la position et de la direction de l'intrus en cas de conflit aérien. De façon similaire, Agrawal et al. [2] recommandent d'utiliser différents 
types d'alertes pour différents éléments du système. Par exemple, la notification de détection d'une victime lors d'une phase de recherche doit être différente d'une alerte sur un niveau bas d'énergie qui nécessite un atterrissage rapide.

4.4.5 Standardiser les commandes et vérifier leurs cohérences. La revue de littérature de Balog et al. [9] suggère que la standardisation des contrôles pour les mini-drones est une clé pour faciliter leur prise en mains. Ceci permettrait d'éviter les erreurs de pilotage quand les télépilotes passent d'un système à un autre [78]. Lorsque plusieurs moyens de pilotage sont disponibles, il est recommandé de faire correspondre les éléments, par exemple de l'interface graphique et de la télécommande, afin de faciliter la reprise de contrôle [2]. Les auteurs suggèrent une vérification systématique de la cohérence lors des opérations. Ils proposent par exemple de déclencher une alarme sur l'interface graphique et de bloquer le décollage tant que les leviers de sécurité ne sont pas correctement positionnés sur la télécommande du pilote.

4.4.6 Faciliter le pilotage et la planification avec la réalité augmentée, la réalité virtuelle ou la 3D. Il est défendu dans plusieurs articles que l'utilisation de la réalité augmentée permet d'améliorer la conscience de la situation des télépilotes. Par exemple, la surimpression pour montrer la géométrie d'un conflit possible [67], voir la mission en 3D et ses différentes zones [15] ou repérer la piste d'atterrissage par mauvais temps [83]. Paterson et al. [85] proposent d'utiliser une vue immersive, en réalité virtuelle, qui améliore la perception de la profondeur et permet de régler le point de vue offert afin de déterminer plus rapidement un chemin adapté pour le drone. Leurs résultats indiquent que la réalité virtuelle permet d'accélérer la création des trajectoires par rapport à une interface en $2 \mathrm{D}$. L'utilisation de la 3D est également recommandée par Besada et al. [15] pour améliorer la planification de trajectoires, notamment la précision dans le placement des différents points dans l'espace. En utilisant des simulations en réalité virtuelle, Thomason et al. [106] montrent qu'une vue adaptative en temps réel, améliore le contrôle et la précision lors du pilotage, en comparaison avec une vue à la première ou à la troisième personne.

4.4.7 Utiliser des représentations cartographiques. Plusieurs articles mettent en évidence le besoin d'utiliser des représentations cartographiques pour le contrôle [47] et pour la surveillance, notamment pour gérer les zones d'interdiction de vol [115]. Selon Primatesta et al. [88], ces représentations sont également appropriées pour réaliser une étude de sécurité. L'utilisation d'une carte de risque au sol, incluant plusieurs couches telle que la densité de population, le facteur de protection en cas de chute, les zones interdites de vol ou encore les obstacles, est utilisée pour l'analyse des risques. Pour la supervision de flotte de drones, Wu et al. [119] suggèrent d'utiliser des cartes tridimensionnelles sur le globe terrestre pour améliorer la précision et la fiabilité des opérateurs.

4.4.8 Faire évoluer progressivement les interfaces. Pour permettre aux contrôleurs aériens d'intégrer les drones en plus des avions dans leurs outils, une approche graduelle est suggérée. Finke et al. [47] suggèrent d'introduire des versions améliorées progressivement les outils actuels pour intégrer les drones, si possible sans impacter les tâches existantes de contrôle du trafic aérien. Ils suggèrent par exemple d'ajouter une représentation symbolique des drones sur l'image radar des contrôleur avec un losange en plus de celle existante sous forme de carré des avions. Pour mesurer la familiarité des contrôleurs aériens avec les nouvelles technologies introduites incluant les drones, Baum et al. [12] suggèrent d'utiliser la métrique du Technical Maturity Level (TML) afin de vérifier l'effet des changements sur la charge de travail.

4.4.9 Permettre la description, la comparaison et l'analyse de missions. Fournir des outils pour élaborer et visualiser un plan de vol complet, comprenant non seulement les trajectoires mais également des annotations sur le risque élevé dans certaines zones, des durées minimales et maximales de vol ou encore des zones d'atterrissage et de décollage des 
drones, est important pour évaluer la sécurité des plans de vol [16]. En plus des ces aspects spatiaux, des informations temporelles sont également importantes pour les risques de conflits avec d'autres appareils, par exemple l'heure et la durée des vols. Pour améliorer la sécurité, Denney et Pai [3] montrent que des outils permettant de comparer et voir les différences entre deux dossiers de sécurité aident les utilisateurs à comprendre l'impact potentiel des changements sur la sécurité. Dans un autre article [2], Agrawal et al. suggèrent que des outils permettant de rejouer des missions déjà effectuées permet une analyse rétrospective bénéfique pour la sécurité des missions futures.

\subsection{Perspectives identifiées}

Dans cette section, nous présentons certaines perspectives de recherches en IHM identifiées dans les articles. Plusieurs articles présentant des expérimentations menées en laboratoire via des simulations [12, 24, 85, 95, 106, 119], des prototypes non réalistes [2] ou des vols d'essais [16,67] préconisent de réaliser des expériences en conditions réelles ou à une échelle plus large afin de valider les résultats obtenus. D’autres expérimentations plus spécifiques sont également évoquées. Par exemple, Agrawal et al. [2], préconisent de réaliser des cas d'études dans lesquels le comportement des drones ne peut pas être facilement expliqué ou compréhensible afin d'étudier la conscience de la situation. Pour Paterson et al. [85], il faut réaliser des études mettant en oeuvre des missions complexes ou avec plusieurs drones pour valider les apports de la réalité virtuelle pour le pilotage de drones.

Pour les articles mettant en oeuvre des adaptations d'interfaces lors de l'utilisation, les perspectives principales sont l'exploitation de plus de sources de données [94], l'emploi d'algorithmes d'apprentissages différents [19, 64, 76] et le test des résultats sur plusieurs scénarios [94]. Bonache-Seco et al. [19] souhaitent également étudier plus en détail l'impact du temps de réaction des opérateurs et l'ajustement de la transparence des éléments de l'interface utilisateur pour améliorer l'adaptation.

Pour aider l'utilisation de systèmes automatisés, Kistan et al. [64] proposent d'étudier l'explicabilité de l'intelligence artificielle utilisée et d'identifier des critères pour juger de la qualité et de la fiabilité de l'explication fournie par la machine ainsi que sa présentation aux utilisateurs ou "Explainable User Interface". Pour permettre aux utilisateurs de contrôler la mission à haut niveau, la possibilité de communiquer les objectifs de la mission aux drones est également envisagée [2]. Baum et al. [12], suggèrent de réaliser des études sur la charge de travail des contrôleurs aérien lorsque des fonctions d'assistance automatisées sont incluses. Plusieurs pistes concernant l'automatisation de certaines tâches sont évoquées. Par exemple, Lim et al. [71] proposent d'étudier les interfaces adaptatives pour la transition d'une opération avec un pilote unique à une opération sans pilote à bord ainsi que les scénarios Extended Visual Lign of Sight (EVLOS), ou en vue étendue, pendant lesquels plusieurs télépilotes se transfèrent les commandes de drones avec l'aide d'observateurs visuels. Denney et Pai $[38,39]$ souhaitent concevoir des outils permettant d'assembler des études de sécurité automatiquement et de développer des interfaces permettant d'explorer ces études.

Faire le lien avec les technologies futures de l'UTM et de contrôle aérien existant est également mentionné par plusieurs articles $[15,16]$. Il s'agit principalement d'ajouter des fonctionnalités dédiées à la gestion des drones dans les interfaces existantes pour la surveillance du trafic aérien.

Concernant la réalisation d'études de sécurité, plusieurs articles indiquent vouloir améliorer les interfaces utilisateurs proposées pour enrichir le type d'informations et de recommandations fournies aux utilisateurs [3, 95], permettre des recherches avancées et des liens entre les multiples documents qui constituent l'analyse de sécurité [38] ou encore des liens entre l'analyse de sécurité et la conception des trajectoires de la mission [39]. 


\section{DISCUSSION}

Notre revue systématique de littérature indique un certain nombre de tendances dans les recherches sur le soutien à la sécurité des opérations de drones. Il y a des tendances fortes dans les axes et les approches de recherche dont nous discutons dans la cette section. Pour plus de clarté, nous structurons cette discussion autour de trois thèmes principaux : les étapes de réalisation des missions, les représentations géographiques pour les analyses de sécurité et les opportunités pour la réalité augmentée et virtuelle. Enfin, nous considérons les limites de validité de notre méthode, requise par la liste de contrôle de la bonne qualité d'une RSL, exigence de PRISMA [80].

\subsection{Disparités entre les étapes et les tâches utilisateurs}

Nos résultats mettent en avant que les étapes de préparation de la mission et surtout de la réalisation concentrent l'attention des recherches ( $91.92 \%$ des articles) et que les étapes de demande d'autorisation, de conception du drone, de l'après-mission ainsi que de la réglementation sont bien moins considérées. Ce déséquilibre n'est pas anodin, car toutes ces autres étapes sont importantes pour assurer la sécurité des missions, d'autant plus que l'évolution de la réglementation va impacter fortement les études de sécurité.

La tendance du trafic de drones étant à l'augmentation, cela rendra plus complexes la production et le traitement d'études de sécurité, tant pour leur réalisation, leur présentation et leur soumission que pour leur vérification, validation et approbation.

Nous pensons qu'il est important de fournir, aux exploitants et aux régulateurs, des outils permettant de faciliter et d'accélérer la réalisation d'études de sécurité et l'obtention d'autorisations spécifiques le cas échéant. Il est important de souligner que, l'European Cockpit Association (ECA), ou l'association européenne des pilotes de ligne, met en garde sur la possibilité que SORA et les STS soient seulement considérés comme un raccourci pour aller plus vite au détriment de la sécurité [45]. Elle suggère que des autorisations basées uniquement sur les déclarations d'exploitants ne doivent pas être permises, pour le moment. Il parait donc important d'accélérer les démarches mais pas le processus de réflexion, qu'il faut au contraire soutenir.

\subsection{Représentations géographiques pour les analyses de sécurité}

Nos résultats indiquent que pour les étapes de préparation et de réalisation de la mission, la plupart des outils disposent de représentations géographiques comme des images radar ou des cartes. Plus en amont, les analyses de sécurité ne se basent pas, sauf exception [88], sur ce genre de représentation mais davantage sur des diagrammes et des éléments textuels plus proches des formalismes utilisés pour raisonner sur les risques. Nous pensons que cette séparation est préjudiciable à la sécurité car cela ne permet pas une transition fluide entre les différentes étapes et fait courir un risque de manque d'information contextuelle sur la géographie du lieu envisagé pour la mission.

Les possibilités offertes par les approches de Détecter et Éviter ainsi que la planification et la replanification qui sont basées sur des problèmes géographiques pourraient aussi servir dès l'élaboration des études de sécurité ou la demande d'autorisation. Ceci permettrait de soutenir les services U-Space envisagée pour l'étape U2 et U3. Lors de l'analyse de sécurité d'une mission nécessitant une autorisation spécifique, l'utilisation d'outils interactifs basés sur des représentations géographiques, permettrait vraisemblablement de faciliter les échanges entre les exploitants et les régulateurs avant la mission, puis d'assurer sa surveillance lors de son déroulement. 


\section{3 Étendre l'usage de la réalité augmentée et réalité virtuelle}

La réalité augmentée ou virtuelle est étudiée par cinq articles [15, 67, 83, 85, 106]. Les informations supplémentaires augmentent la conscience de la situation des télépilotes et peuvent constituer une aide effective au pilotage. La réalité virtuelle apporte plus de facilité lors du pilotage ainsi qu'une mise en oeuvre plus rapides des corrections lors de la planification de trajectoires. Nous suggérons alors de s'intéresser à l'utilisation de ces technologies pour le pilotage et la préparation de mission mais aussi d'étudier les avantages qu'elles pourraient amener pour les autres tâches : analyse de sécurité, demande d'autorisation, contrôle et surveillance.

\subsection{Limites sur la validité}

L'inaccessibilité de certains articles, le référencement sous d'autres mots-clés ou dans d'autres bases de données que celles utilisées, notez par exemple que Scopus n'a pas été consulté, peuvent impacter le processus de sélection des articles. Celui-ci a été suivi aussi rigoureusement que possible mais peut présenter des biais. Dans le cas où certains articles abordaient plusieurs approches potentiellement proches pour le soutien à la sécurité, un des auteurs a du effectuer le classement. Par exemple, les notions de Détecter \& Éviter et de planification de trajectoires peuvent être relativement similaires puisque l'évitement consiste à proposer une nouvelle trajectoire. Cette classification a pu influencer les résultats. L'ajout d'articles issus d'une première itération de la RSL mais avec des mots clés très ciblés dans le titre nous a semblé pertinente mais peut modifier le nombre total d'articles considérés, l'opération de filtrage et la répartition finale. Enfin, pour certains articles, bien que certaines réponses au QQOQCCP ne figuraient pas explicitement dans l'article, les explications, les contraintes, la façon de procéder aux simulations et aux tests ont parfois permis de compléter les informations. Selon l'extracteur, le filtrage et la classification peuvent varier. La vérification par un second extracteur nous permet de minimiser les éventuels biais de sélection et de classification.

\section{CONCLUSION}

Dans cet article, nous nous intéressons au soutien à la sécurité des opérations de drones. Nous avons décrit le système de drone civil et synthétisé la réglementation européenne sur les drones. Nous avons réalisé une revue systématique de littérature en utilisant une déclinaison de la méthode QQOQCCP pour classifier le soutien à la sécurité des missions de drones. Cela nous a permis d'établir l'état de l'art sur ce sujet, et de dégager notamment huit grandes catégories d'approche : le Détecter \& Éviter, les IHM et facteurs humains, l'intégrité de l'aéronef, les analyses de sécurité, la planification de trajectoires, la gestion du trafic de drones, la cyber-sécurité et le géorepérage. Nous avons identifié les types de contributions de l'IHM et FH pour les tâches de surveillance, de pilotage, de préparation de missions, d'analyses de sécurité, de contrôle et de demande d'autorisation. Notre avons analysé les recommandations formulées par les articles pour maximiser la sécurité lors de la conception d'interfaces et d'interactions ainsi que les perspectives de recherche évoquées. Ces travaux fournissent une base pour comprendre et décrire le domaine du soutien à la sécurité des missions de drones. Nous espérons que ces travaux permettront aux concepteurs de systèmes de drones d'utiliser et d'adapter des solutions existantes pour l'aide à la gestion de la sécurité pour différents types de missions. Nous pensons que les pistes de recherches identifiées, telles que l'étude des phases d'analyse de sécurité ou de demande d'autorisation, l'utilisation de représentations géographiques lors de ces phases ainsi que les possibilités de la réalité augmentée et de la réalité virtuelle, peuvent guider les efforts de la communauté IHM dans ce domaine. 


\section{REMERCIEMENTS}

Nous remercions Jim Sharples et Yannick Jestin de l'Ecole Nationale de l'Aviation Civile (ENAC) pour leur apports sur la réglementation et les systèmes de drone. Ce travail a été partiellement financé par Agence de l'Innovation de Défense (AID) du ministère de la Défense (Projet CONCORDE N²019 65 0090004707501).

\section{RÉFÉRENCES}

[1] Shubhani Aggarwal and Neeraj Kumar. 2020. Path planning techniques for unmanned aerial vehicles : A review, solutions, and challenges. Computer Communications 149 (2020), 270 - 299. https://doi.org/10.1016/j.comcom.2019.10.014

[2] Ankit Agrawal, Sophia J. Abraham, Benjamin Burger, Chichi Christine, Luke Fraser, John M. Hoeksema, Sarah Hwang, Elizabeth Travnik, Shreya Kumar, Walter Scheirer, Jane Cleland-Huang, Michael Vierhauser, Ryan Bauer, and Steve Cox. 2020. The Next Generation of Human-Drone Partnerships : Co-Designing an Emergency Response System. (2020), 1-13. https://doi.org/10.1145/3313831.3376825

[3] Ankit Agrawal, Seyedehzahra Khoshmanesh, Michael Vierhauser, Mona Rahimi, Jane Cleland-Huang, and Robyn Lutz. 2019. Leveraging Artifact Trees to Evolve and Reuse Safety Cases. (2019), 1222-1233. https://doi.org/10.1109/ICSE.2019.00124

[4] Azza Allouch, Anis Koubaa, Mohamed Khalgui, and Tarek Abbes. 2019. Qualitative and Quantitative Risk Analysis and Safety Assessment of Unmanned Aerial Vehicles Missions Over the Internet. IEEE ACCESS 7 (2019), 53392-53410. https://doi.org/10.1109/ACCESS.2019.2911980

[5] Riham Altawy and Amr M. Youssef. 2016. Security, Privacy, and Safety Aspects of Civilian Drones : A Survey. ACM Trans. Cyber-Phys. Syst. 1, 2, Article 7 (Nov. 2016), 25 pages. https://doi.org/10.1145/3001836

[6] Riham Altawy and Amr M. Youssef. 2016. Security, Privacy, and Safety Aspects of Civilian Drones : A Survey. ACM Trans. Cyber-Phys. Syst. 1, 2, Article 7 (nov 2016), 25 pages. https://doi.org/10.1145/3001836

[7] Adnan Ashraf, Amin Majd, and Elena Troubitsyna. 2017. Towards a Realtime, Collision-Free Motion Coordination and Navigation System for a UAV Fleet. , Article 11 (2017), 9 pages. https://doi.org/10.1145/3123779.3123805

[8] Rémi Bachelet. 2012. Méthode de Résolution de Problèmes : QQOQCP. http://rb.ec-lille.fr/1/Qualite/Qualite_QQOQCCP.pdf.

[9] Clint R. Balog, Brent A. Terwilliger, Dennis A. Vincenzi, and David Carl Ison. 2017. Examining Human Factors Challenges of Sustainable Small Unmanned Aircraft System (sUAS) Operations. In Advances in Human Factors in Robots and Unmanned Systems, Savage-Knepshield P. and Chen J. (Eds.). Springer.

[10] Jose de Sousa Barros, Thyago Oliveira Freitas, Vivek Nigam, and Alisson V. Brito. 2017. Analysis of design strategies for unmanned aerial vehicles using co-simulation. DESIGN AUTOMATION FOR EMBEDDED SYSTEMS 21, 3-4, SI (DEC 2017), 157-172. https://doi.org/10.1007/s10617-017-9190-z

[11] Eleonora Bassi. 2020. From Here to 2023 : Civil Drones Operations and the Setting of New Legal Rules for the European Single Sky. JOURNAL OF INTELLIGENT \& ROBOTIC SYSTEMS (2020). https://doi.org/10.1007/s10846-020-01185- 1

[12] Derick Moreira Baum, Euclides Carlos Pinto Neto, Jorge Rady De Almeida, Joao Batista Camargo, and Paulo Sergio Cugnasca. 2020. A MindsetBased Evolution of Unmanned Aircraft System (UAS) Acceptance Into the National Airspace System (NAS). IEEE ACCESS 8 (2020), $30938-30952$. https://doi.org/10.1109/ACCESS.2019.2952973

[13] Amir Behjat, Steve Paul, and Souma Chowdhury. 2019. Learning reciprocal actions for cooperative collision avoidance in quadrotor unmanned aerial vehicles. ROBOTICS AND AUTONOMOUS SYSTEMS 121 (NOV 2019). https://doi.org/10.1016/j.robot.2019.103270

[14] Fethi Belkhouche. 2017. Reactive Optimal UAV Motion Planning in a Dynamic World. Robot. Auton. Syst. 96, C (oct 2017), 114-123. https: //doi.org/10.1016/j.robot.2017.07.006

[15] Juan A Besada, Luca Bergesio, Iván Campaña, Diego Vaquero-Melchor, Jaime López-Araquistain, Ana M Bernardos, and Jose R Casar. 2018. Drone mission definition and implementation for automated infrastructure inspection using airborne sensors. Sensors 18, 4 (2018), 1170.

[16] Juan A Besada, Iván Campaña, Luca Bergesio, Ana M. Bernardos, and Gonzalo de Miguel. 2019. Drone Flight Planning for Safe Urban Operations : UTM Requirements and Tools. In 2019 IEEE International Conference on Pervasive Computing and Communications Workshops (PerCom Workshops). 924-930.

[17] Zachary Birnbaum, Andrey Dolgikh, Victor Skormin, Edward O’Brien, Daniel Muller, and Christina Stracquodaine. 2016. Unmanned Aerial Vehicle Security Using Recursive Parameter Estimation. FOURNAL OF INTELLIGENT \& ROBOTIC SYSTEMS 84, 1-4, SI (DEC 2016), 107-120. https://doi.org/10.1007/s10846-015-0284-1

[18] Mark Blanks. 2014. UAS Applications. In Introduction to Unmanned Aircraft Systems - Second Edition, Richard K. Barnhardt, Stephen B. Hottman, Douglas M. Marshall, and Eric Shappee (Eds.). Taylor and Francis, Chapter 2.

[19] Juan A. Bonache-Seco, José A. Lopez-Orozco, Eva Besada-Portas, and José L. Risco-Martín. 2019. ART-GCS : An Adaptive Real-Time Multi-Agent Ground Control Station. , Article 13 (2019), 12 pages.

[20] Thierry Bontems. 2011. Process management - "From theory to practice". In 9ème Ecole Inter-organismes "Qualité en Recherche et en Enseignement Supérieur", 2011/09/7-9, Montpellier (France) (Les cahiers de l'école qualité en recherche et en enseignement supérieur, 2), Montpellier [France] : CIHEAM-IAMM (Ed.). QUARES, Montpellier, France, 155. https://halshs.archives-ouvertes.fr/halshs-01402572

[21] Hana Bouafif, Faouzi Kamoun, and Farkhund Iqbal. 2020. Towards a Better Understanding of Drone Forensics : A Case Study of Parrot AR Drone 2.0. INTERNATIONAL JOURNAL OF DIGITAL CRIME AND FORENSICS 12, 1 (JAN-MAR 2020), 35-57. https://doi.org/10.4018/IJDCF.2020010103 
[22] Mark Bowkett, Kary Thanapalan, and Ewen Constant. 2018. Failure Detection of Composites with Control System Corrective Response in Drone System Applications. COMPUTERS 7, 2 (JUN 2018). https://doi.org/10.3390/computers7020023

[23] Joshua Brungardt and Richard K. Barnhardt. 2014. The "System" in UAS. In Introduction to Unmanned Aircraft Systems - Second Edition, Richard K. Barnhardt, Stephen B. Hottman, Douglas M. Marshall, and Eric Shappee (Eds.). Taylor and Francis, Chapter 3.

[24] Ivan Campaña, Luca Bergesio, Juan Alberto Besada Portas, and Gonzalo de Miguel. 2019. Air Tracking and Monitoring For Unmanned Aircraft Traffic Management. In 2019 Integrated Communications, Navigation and Surveillance Conference (ICNS). 1-9.

[25] Carlos Capitán, Jesus Capitán, Ángel Castaño, and Anibal Ollero. 2019. Risk Assessment based on SORA Methodology for a UAS Media Production Application. In 2019 International Conference on Unmanned Aircraft Systems (ICUAS). 451-459.

[26] Giovanna Castellano, Ciro Castiello, Corrado Mencar, and Gennaro Vessio. 2020. Crowd Detection in Aerial Images Using Spatial Graphs and Fully-Convolutional Neural Networks. IEEE ACCESS 8 (2020), 64534-64544. https://doi.org/10.1109/ACCESS.2020.2984768

[27] Yuqing Chen, Gaoxiang Zhang, Yan Zhuang, and Huosheng Hu. 2019. Autonomous Flight Control for Multi-Rotor UAVs Flying at Low Altitude. IEEE ACCESS 7 (2019), 42614-42625. https://doi.org/10.1109/ACCESS.2019.2908205

[28] Jung Hee Cheon, Kyoohyung Han, Seong-Min Hong, Hyoun Jin Kim, Junsoo Kim, Suseong Kim, Hosung Seo, Hyungbo Shim, and Yongsoo Song 2018. Toward a Secure Drone System : Flying With Real-Time Homomorphic Authenticated Encryption. IEEE ACCESS 6 (2018), 24325-24339. https://doi.org/10.1109/ACCESS.2018.2819189

[29] Jane Cleland-Huang, Michael Vierhauser, and Sean Bayley. 2018. Dronology : An Incubator for Cyber-Physical System Research. arXiv preprint arXiv :1804.02423 (2018).

[30] Commission de l'Union Européenne. 2019. Règlement d'exécution (UE) 2019/947 de la Commission du 24 mai 2019 concernant les règles et procédures applicables à l'exploitation d'aéronefs sans équipage à bord. https://eur-lex.europa.eu/eli/reg_del/2019/947/oj?locale=fr.

[31] Commission de l'Union Européenne. 2019. Règlement délégué (UE) 2019/945 de la Commission du 12 mars 2019 relatif aux systèmes d'aéronefs sans équipage à bord et aux exploitants, issus de pays tiers, de systèmes d'aéronefs sans équipage à bord. https://eur-lex.europa.eu/eli/reg_del/2019/945/oj?locale=fr.

[32] Federico Corraro, Gianluca Corraro, Edoardo Filippone, and Leopoldo Verde. 2019. A Multi-Intruder Robust Collision Avoidance System for Integration of Unmanned Vehicles in Civil Transport. (2019), 2338-2343. https://doi.org/10.1109/SMC.2019.8914430

[33] Alan Cullen, Bill Williams, Elisa Bertino, Saritha Arunkumar, Erisa Karafili, and Emil Lupu. 2017. Mission Support for Drones : A Policy Based Approach. (2017), 7-12. https://doi.org/10.1145/3086439.3086444

[34] Jesimar da Silva Arantes, Márcio da Silva Arantes, Claudio Fabiano Motta Toledo, Onofre Trindade Júnior, and Brian C. Williams. 2017. An Embedded System Architecture Based on Genetic Algorithms for Mission and Safety Planning with UAV. (2017), 1049-1056. https://doi.org/10. $1145 / 3071178.3071302$

[35] Egidio D'Amato, Massimiliano Mattei, and Immacolata Notaro. 2020. Distributed Reactive Model Predictive Control for Collision Avoidance of Unmanned Aerial Vehicles in Civil Airspace. JOURNAL OF INTELLIGENT \& ROBOTIC SYSTEMS 97, 1 (JAN 2020), 185-203. https://doi.org/10. 1007/s10846-019-01047-5

[36] Joao Luiz de Castro Fortes, Rafael Fraga, and Kenny Martin. 2016. An Approach for Safety Assessment in UAS Operations Applying Stochastic Fast-Time Simulation with Parameter Variation. (2016), 1860-1871.

[37] Ewen Denney and Ganesh Pai. 2014. Automating the Assembly of Aviation Safety Cases. IEEE TRANSACTIONS ON RELIABILITY 63, 4 (DEC 2014), 830-849. https://doi.org/10.1109/TR.2014.2335995

[38] Ewen Denney and Ganesh Pai. 2018. Tool support for assurance case development. AUTOMATED SOFTWARE ENGINEERING 25, 3 (SEP 2018), 435-499. https://doi.org/10.1007/s10515-017-0230-5

[39] Ewen Denney, Ganesh Pai, and Iain Whiteside. 2017. Model-Driven Development of Safety Architectures. (2017), 156-166. https://doi.org/10.1109/ MODELS.2017.27

[40] Carmelo Di Franco and Giorgio Buttazzo. 2016. Coverage Path Planning for UAVs Photogrammetry with Energy and Resolution Constraints. JOURNAL OF INTELLIGENT \& ROBOTIC SYSTEMS 83, 3-4, SI (SEP 2016), 445-462. https://doi.org/10.1007/s10846-016-0348-x

[41] Kay Dickersin and Tianjin Li. 2019. Introduction to Systematic Reviews and Meta-Analysis. https://www.coursera.org/learn/systematicreview/lecture/eCyR3/lecture-1a-introduction-to-systematic-reviews.

[42] Direction Générale de l'Aviation Civile. 2018. Guide des Aéronefs circulant sans personnes à bord : ACTIVITÉS PARTICULIÈRES. Periodic Reports.

[43] Yanshuang Du, Xuejun Zhang, and Qi Gu. 2019. Adaptive Separation Thresholds for Self-Separation of Unmanned Aircraft System in Dynamic Airspace. IEEE ACCESS 7 (2019), 141817-141825. https://doi.org/10.1109/ACCESS.2019.2941220

[44] Yanshuang Du, Xuejun Zhang, and Zunli Nie. 2019. A Real-Time Collision Avoidance Strategy in Dynamic Airspace Based on Dynamic Artificial Potential Field Algorithm. IEEE ACCESS 7 (2019), 169469-169479. https://doi.org/10.1109/ACCESS.2019.2953946

[45] European Cockpit Association. 2019. Specific Operations Risk Assessment (SORA). https://www.eurocockpit.be/positions-publications/specificoperations-risk-assessment-sora.

[46] Baoan Fan and Guigen Zeng. 2019. Obstacle Avoidance System Design Based on Quad - Rotor UAV. In Proceedings of the 2019 4th International Conference on Robotics, Control and Automation (Guangzhou, China) (ICRCA 2019). Association for Computing Machinery, New York, NY, USA, 144-147. https://doi.org/10.1145/3351180.3351212 
[47] Michael Finke, Malte-Levin Jauer, and Kathleen Muth. 2018. Deriving Support Functions for Radar Approach Controllers Confronted with Mixed Manned and Unmanned Air Traffic. In Deutscher Luft- und Raunfahrtkongress 2018 (Friedrichshafen, Germany).

[48] Qixi Fu, Xiaolong Liang, Jiaqiang Zhang, Duo Qi, and Xiujun Zhang. 2019. Design and Experiment of Autonomous Flight UAS Geofence Algorithm. In Proceedings of the 2nd International Conference on Computer Science and Software Engineering (Xi'an, China) (CSSE 2019). Association for Computing Machinery, New York, NY, USA, 137-143. https://doi.org/10.1145/3339363.3339381

[49] Zhangjie Fu, Yuanhang Mao, Daojing He, Jingnan Yu, and Guowu Xie. 2019. Secure Multi-UAV Collaborative Task Allocation. IEEE ACCESS 7 (2019), 35579-35587. https://doi.org/10.1109/ACCESS.2019.2902221

[50] Jeremi Gancet, Gautier Hattenberger, Rachid Alami, and Simon Lacroix. 2005. Task planning and control for a multi-UAV system : Architecture and algorithms. Proc. IROS Conference, 1017 - 1022. https://doi.org/10.1109/IROS.2005.1545217

[51] Jérémie Garcia, Anke Brock, Nicolas Saporito, Gautier Hattenberger, Xavier Paris, Michel Gorraz, and Yannick Jestin. 2019. Designing human-drone interactions with the Paparazzi UAV System. In 1st International Workshop on Human-Drone Interaction. Ecole Nationale de l'Aviation Civile [ENAC], Glasgow, United Kingdom. https://hal.archives-ouvertes.fr/hal-02128390

[52] Jose Antonio Garcia-Pulido, Gonzalo Pajares, Sebastian Dormido, and Jesus Manuel de la Cruz. 2017. Recognition of a landing platform for unmanned aerial vehicles by using computer vision-based techniques. EXPERT SYSTEMS WITH APPLICATIONS 76 (JUN 15 2017), $152-165$. https://doi.org/10.1016/j.eswa.2017.01.017

[53] Ricardo A. V. Gimenes, Lucio F. Vismari, Valter F. Avelino, Joao B. Camargo, Jr., Jorge R. de Almeida, Jr., and Paulo S. Cugnasca. 2014. Guidelines for the Integration of Autonomous UAS into the Global ATM. FOURNAL OF INTELLIGENT \& ROBOTIC SYSTEMS 74, 1-2, SI (APR 2014), 465-478. https://doi.org/10.1007/s10846-013-9945-0

[54] Sergio Alejandro Gómez, Anca Goron, Adrian Groza, and Ioan Alfred Letia. 2016. Assuring Safety in Air Traffic Control Systems with Argumentation and Model Checking. Expert Syst. Appl. 44, C (feb 2016), 367-385. https://doi.org/10.1016/j.eswa.2015.09.027

[55] Veronica Gonzalez, Concepcion A. Monje, Luis Moreno, and Carlos Balaguer. 2017. UAVs mission planning with flight level constraint using Fast Marching Square Method. ROBOTICS AND AUTONOMOUS SYSTEMS 94 (AUG 2017), 162-171. https://doi.org/10.1016/j.robot.2017.04.021

[56] Mikhail Gorobetz, Leonids Ribickis, Anatoly Levchenkov, and Anna Beinarovica. 2019. Machine Learning Algorithm of Immune Neuro-Fuzzy AntiCollision Embedded System for Autonomous Unmanned Aerial Vehicles Team. , Article 25 (2019), 8 pages. https://doi.org/10.1145/3309772.3309797

[57] Dingfei Guo, Maiying Zhong, Hongquan Ji, Yang Liu, and Rui Yang. 2018. A hybrid feature model and deep learning based fault diagnosis for unmanned aerial vehicle sensors. NEUROCOMPUTING 319 (NOV 30 2018), 155-163. https://doi.org/10.1016/j.neucom.2018.08.046

[58] Rongxiao Guo, Buhong Wang, and Jiang Weng. 2020. Vulnerabilities and Attacks of UAV Cyber Physical Systems. In Proceedings of the 2020 International Conference on Computing, Networks and Internet of Things (Sanya, China) (CNIOT2020). Association for Computing Machinery, New York, NY, USA, 8-12. https://doi.org/10.1145/3398329.3398331

[59] Daojing He, Hong Liu, Sammy Chan, and Mohsen Guizani. 2019. How to Govern the Non-Cooperative Amateur Drones? IEEE NETWORK 33, 3 (MAY-JUN 2019), 184-189. https://doi.org/10.1109/MNET.2019.1800156

[60] Florence Ho, Ruben Geraldes, Artur Goncalves, Marc Cavazza, and Helmut Prendinger. 2018. Simulating Shared Airspace for Service UAVs with Conflict Resolution. (2018), 2192-2194.

[61] ICAO. 2015. Manual on Remotely Piloted Aircraft Systems (RPAS) - Doc 10039 - AN/507. International Civil Aviation Organization.

[62] Uluhan C. Kaya, Atilla Dogan, and Manfred Huber. 2019. A Utility-Based Path Planning for Safe UAS Operations with a Task-Level Decision-Making Capability. (2019), 1227-1233. https://doi.org/10.1109/SMC.2019.8914226

[63] Do-Yup Kim and Jang-Won Lee. 2020. Joint Mission Assignment and Topology Management in the Mission-Critical FANET. IEEE INTERNET OF THINGS JOURNAL 7, 3 (MAR 2020), 2368-2385. https://doi.org/10.1109/JIOT.2019.2958130

[64] Trevor Kistan, Alessandro Gardi, and Roberto Sabatini. 2018. Machine Learning and Cognitive Ergonomics in Air Traffic Management : Recent Developments and Considerations for Certification. Aerospace Application of Multiagent Systems and Artificial Intelligence Techniques in Aviation (2018).

[65] Parimal Kopardekar, Joseph Rios, Thomas Prevot, Marcus Johnson, Jaewoo Jung, and John E. Robinson III. 2016. Unmanned Aircraft System Traffic Management (UTM) Concept of Operations. AIAA Aviation 16th AIAA Aviation Technology, Integration, and Operations Conference (june 2016).

[66] Anders la Cour-Harbo. 2019. Quantifying Risk of Ground Impact Fatalities for Small Unmanned Aircraft. FOURNAL OF INTELLIGENT \& ROBOTIC SYSTEMS 93, 1-2, 2, SI (FEB 2019), 367-384. https://doi.org/10.1007/s10846-018-0853-1

[67] Patrick Le Blaye and Claude Le Tallec. 2018. Low level traffic monitoring : RPAS concept of operation and development of a ground based system. In ICAS 2018. BELO HORIZONTE, Brazil. https://hal.archives-ouvertes.fr/hal-02000595

[68] Claude Le Tallec and Patrick Le Blaye. 2017. Low Level RPAS Traffic Identification and Management. In 8th European Conference for Aeronautics and Space Sciences EUCASS (Milan, Italy).

[69] Sang Eon Lee, Jae-Wook Jung, Youngjun Choi, Yoon-Jin Yoon, and Jung-Wuk Hong. 2019. Unmanned Aerial Vehicle Impacts on Heat-Strengthened Glass. IEEE ACCESS 7 (2019), 104269-104278. https://doi.org/10.1109/ACCESS.2019.2930277

[70] Shidong Li, Huihua Zhou, Jia Hu, Qing Ai, and Chao Cai. 2015. A fast path planning approach for unmanned aerial vehicles. CONCURRENCY AND COMPUTATION-PRACTICE \& EXPERIENCE 27, 13, SI (SEP 10 2015), 3446-3460. https://doi.org/10.1002/cpe.3291

[71] Yixiang Lim, Alessandro Gardi, Roberto Sabatini, Subramanian Ramasamy, and Trevor Kistan. 2018. Avionics Human-Machine Interfaces and Interactions for Manned and Unmanned Aircraft. Progress in Aerospace Science 102 (2018). 
[72] Yixiang Lim, Subramanian Ramasamy, Alessandro Gardi, Trevor Kistan, and Roberto Sabatini. 2018. Cognitive Human-Machine Interfaces and Interactions for Unmanned Aircraft. Journal of Intelligent Robot Systems 91 (2018).

[73] Chin E. Lin and Ya-Hsien Lai. 2015. Quasi-ADS-B Based UAV Conflict Detection and Resolution to Manned Aircraft. FOURNAL OF ELECTRICAL AND COMPUTER ENGINEERING (2015). https://doi.org/10.1155/2015/297859

[74] Alexandros Lioulemes, Georgios Galatas, Vangelis Metsis, Gian Luca Mariottini, and Fillia Makedon. 2014. Safety Challenges in Using AR.Drone to Collaborate with Humans in Indoor Environments. In Proceedings of the 7th International Conference on PErvasive Technologies Related to Assistive Environments (Rhodes, Greece) (PETRA '14). Association for Computing Machinery, New York, NY, USA, Article 33, 4 pages. https: //doi.org/10.1145/2674396.2674457

[75] Jia Liu, Xiaolin Qin, Baolian Qi, and Xiaoli Cui. 2020. 3D ONLINE PATH PLANNING OF UAV BASED ON IMPROVED DIFFERENTIAL EVOLUTION AND MODEL PREDICTIVE CONTROL. INTERNATIONAL JOURNAL OF INNOVATIVE COMPUTING INFORMATION AND CONTROL 16, 1 (FEB 2020), 315-329. https://doi.org/10.24507/ijicic.16.01.315

[76] Jonas Lundberg, Magnus Bång, Jimmy Johansson, Ali Cheaitou, Billy Josefsson, and Zain Tahboub. 2019. Human-in-the-loop AI : Requirements on future (unified) air traffic management systems. In 2019 IEEE/AIAA 38th Digital Avionics Systems Conference (DASC). 1-9.

[77] Imen Mahjri, Amine Dhraief, Abdelfettah Belghith, and Ahmad S. AlMogren. 2018. SLIDE : A Straight Line Conflict Detection and Alerting Algorithm for Multiple Unmanned Aerial Vehicles. IEEE TRANSACTIONS ON MOBILE COMPUTING 17, 5 (MAY 12018 ), 1190-1203. https: //doi.org/10.1109/TMC.2017.2750144

[78] Jason S McCarley and Christopher D Wickens. 2005. Human factors implications of UAVs in the national airspace. (2005).

[79] Ministère de la Transition Ecologique et Solidaire. 2020. Drones - Usages professionnels. https://www.ecologique-solidaire.gouv.fr/drones-usagesprofessionnels.

[80] David Moher, Alessandro Liberati, Jennifer Tetzlaff, and Douglas G. Altman. 2009. Preferred Reporting Items for Systematic Reviews and MetaAnalyses : The PRISMA Statement. Annals of Internal Medicine 151, 4 (Aug. 2009), 264-269. https://doi.org/10.7326/0003-4819-151-4-20090818000135 Publisher : American College of Physicians.

[81] Sebti Mouelhi, Mohamed-Emine Laarouchi, Daniela Cancila, and Hakima Chaouchi. 2019. Predictive Formal Analysis of Resilience in Cyber-Physical Systems. IEEE ACCESS 7 (2019), 33741-33758. https://doi.org/10.1109/ACCESS.2019.2903153

[82] César A. Muñoz, Aaron Dutle, Anthony Narkawicz, and Jason Upchurch. 2016. Unmanned Aircraft Systems in the National Airspace System : A Formal Methods Perspective. ACM SIGLOG News 3, 3 (aug 2016), 67-76. https://doi.org/10.1145/2984450.2984459

[83] N. Nagarani, P. Venkatakrishnan, and Narayanam Balaji. 2020. Unmanned Aerial vehicles runway landing system with efficient target detection by using morphological fusion for military surveillance system. COMPUTER COMMUNICATIONS 151 (FEB 1 2020), 463-472. https://doi.org/10.1016/j. comcom.2019.12.039

[84] Chaofeng Pan, Haotian Shan, Xianbin Cao, Xuelong Li, and Dapeng Wu. 2017. Leveraging Spatial Context Disparity for Power Line Detection. COGNITIVE COMPUTATION 9, 6 (DEC 2017), 766-779. https://doi.org/10.1007/s12559-017-9488-y

[85] Jesse Rawlins Paterson, Jiwoong Han, Tom Cheng, Paxtan Huish Laker, David Livingston McPherson, Joseph Menke, and Allen Y Yang. 2019. Improving Usability, Efficiency, and Safety of UAV Path Planning through a Virtual Reality Interface. In Symposium on Spatial User Interaction (New Orleans, LA, USA) (SUI '19). Association for Computing Machinery, New York, NY, USA, Article 28, 2 pages. https://doi.org/10.1145/3357251.3362742

[86] Timothy Patterson, Sally McClean, Philip Morrow, Gerard Parr, and Chunbo Luo. 2014. Timely autonomous identification of UAV safe landing zones. IMAGE AND VISION COMPUTING 32, 9 (SEP 2014), 568-578. https://doi.org/10.1016/j.imavis.2014.06.006

[87] Joshua Peschel and Robin Murphy. 2013. On the Human-Machine Interaction of Unmanned Aerial System Mission Specialists. Human-Machine Systems, IEEE Transactions on 43 (01 2013), 53-62. https://doi.org/10.1109/TSMCC.2012.2220133

[88] Stefano Primatesta, Alessandro Rizzo, and Anders la Cour-Harbo. 2020. Ground Risk Map for Unmanned Aircraft in Urban Environments. JOURNAL OF INTELLIGENT \& ROBOTIC SYSTEMS 97, 3-4 (MAR 2020), 489-509. https://doi.org/10.1007/s10846-019-01015-z

[89] Xin Qi, Juntong Qi, Didier Theilliol, Dalei Song, Youmin Zhang, and Jianda Han. 2016. Self-Healing Control Design under Actuator Fault Occurrence on Single-rotor Unmanned Helicopters. FOURNAL OF INTELLIGENT \& ROBOTIC SYSTEMS 84, 1-4, SI (DEC 2016), 21-35. https: //doi.org/10.1007/s10846-016-0341-4

[90] Xin Qin and Jyotirmoy V. Deshmukh. 2019. Preview of Predictive Monitoring for Signal Temporal Logic with Probabilistic Guarantees. (2019), 19-21. https://doi.org/10.1145/3313149.3313370

[91] Huaxin Qiu and Haibin Duan. 2020. A multi-objective pigeon-inspired optimization approach to UAV distributed flocking among obstacles. INFORMATION SCIENCES 509 (JAN 2020), 515-529. https://doi.org/10.1016/j.ins.2018.06.061

[92] Subramanian Ramasamy, Roberto Sabatini, and Alessandro Gardi. 2018. A Unified Analytical Framework for Aircraft Separation Assurance and UAS Sense-and-Avoid. Fournal of Intelligent \& Robotic Systems 91, 3 (2018), 735-754. https://doi.org/10.1007/s10846-017-0661-z

[93] Mariana Rodrigues and Kalinka Regina Lucas Jaquie Castelo Branco. 2020. Cloud-SPHERE : Towards Secure UAV Service Provision. FOURNAL OF INTELLIGENT \& ROBOTIC SYSTEMS 97, 1 (JAN 2020), 249-268. https://doi.org/10.1007/s10846-019-01046-6

[94] Victor Rodriguez-Fernandez, Hector D. Menendez, and David Camacho. 2017. A study on performance metrics and clustering methods for analyzing behavior in UAV operations. FOURNAL OF INTELLIGENT \& FUZZY SYSTEMS 32, 2 (2017), 1307-1319. https://doi.org/10.3233/JIFS-169129

[95] Clayton D. Rothwell and Michael J. Patzek. 2019. An Interface for Verification and Validation of Unmanned Systems Mission Planning : Communicating Mission Objectives and Constraints. IEEE TRANSACTIONS ON HUMAN-MACHINE SYSTEMS 49, 6 (DEC 2019), 642-651. https: //doi.org/10.1109/THMS.2019.2945618 
[96] José Rufino. 2016. Towards Integration of Adaptability and Non-Intrusive Runtime Verification in Avionic Systems. SIGBED Rev. 13, 1 (mar 2016), 60-65. https://doi.org/10.1145/2907972.2907981

[97] Davide Di Ruscio, Ivano Malavolta, Patrizio Pelliccione, and Massimo Tivoli. 2016. Automatic Generation of Detailed Flight Plans from High-Level Mission Descriptions. (2016), 45-55. https://doi.org/10.1145/2976767.2976794

[98] Mohammad Fattahi Sani, Maryam Shoaran, and Ghader Karimian. 2019. Automatic landing of a low-cost quadrotor using monocular vision and Kalman filter in GPS-denied environments. TURKISH JOURNAL OF ELECTRICAL ENGINEERING AND COMPUTER SCIENCES 27, 3 (2019), 1821-1838. https://doi.org/10.3906/elk-1809-204

[99] Google Scholar. [n.d.]. Top publications ranking in Human Computer Interactions. https://scholar.google.es/citations?view_op=top_venues\&hl= en\&vq=eng_humancomputerinteraction. Accessed : 2021-01-26.

[100] SESAR JU. 2017. U-space Blueprint. Single European Sky ATM Research Joint Undertaking.

[101] SESAR JU. 2020. U-SPACE. https://www.sesarju.eu/U-space.

[102] Daniel Baraldi Sesso, Lucio F. Vismari, Antonio Vieira da Silva Neto, Paulo S. Cugnasca, and Joao B. Camargo. 2016. An Approach to Assess the Safety of ADS-B-based Unmanned Aerial Systems : Data Integrity as a Safety Issue. FOURNAL OF INTELLIGENT \& ROBOTIC SYSTEMS 84, 1-4, SI (DEC 2016), 621-638. https://doi.org/10.1007/s10846-015-0321-0

[103] Ashutosh Singandhupe, Hung Manh La, and David Feil-Seifer. 2018. Reliable Security Algorithm for Drones Using Individual Characteristics From an EEG Signal. IEEE ACCESS 6 (2018), 22976-22986. https://doi.org/10.1109/ACCESS.2018.2827362

[104] Mia N. Stevens, Hossein Rastgoftar, and Ella M. Atkins. 2019. Geofence Boundary Violation Detection in 3D Using Triangle Weight Characterization with Adjacency. FOURNAL OF INTELLIGENT \& ROBOTIC SYSTEMS 95, 1 (JUL 2019), 239-250. https://doi.org/10.1007/s10846-018-0930-5

[105] Dante Tezza and Marvin Andujar. 2019. The State-of-the-Art of Human-Drone Interaction : A Survey. IEEE Access 7 (2019).

[106] John Thomason, Photchara Ratsamee, Jason Orlosky, Kiyoshi Kiyokawa, Tomohiro Mashita, Yuki Uranishi, and Haruo Takemura. 2019. A Comparison of Adaptive View Techniques for Exploratory 3D Drone Teleoperation. ACM Trans. Interact. Intell. Syst. 9, 2-3, Article 17 (mar 2019), 19 pages. https://doi.org/10.1145/3232232

[107] Yifan Tian, Jiawei Yuan, and Houbing Song. 2019. Efficient privacy-preserving authentication framework for edge-assisted Internet of Drones. JOURNAL OF INFORMATION SECURITY AND APPLICATIONS 48 (OCT 2019). https://doi.org/10.1016/j.jisa.2019.06.010

[108] David Tranfield, David Denyer, and Palminder Smart. 2003. Towards a Methodology for Developing Evidence-Informed Management Knowledge by Means of Systematic Review*. British fournal of Management 14 (2003).

[109] Hanif Ullah, Nithya Gopalakrishnan Nair, Adrian Moore, Chris Nugent, Paul Muschamp, and Maria Cuevas. 2019. 5G Communication : An Overview of Vehicle-to-Everything, Drones, and Healthcare Use-Cases. IEEE ACCESS 7 (2019), 37251-37268. https://doi.org/10.1109/ACCESS.2019.2905347

[110] Hector Usach, Juan A. Vila, Christoph Torens, and Florian Adolf. 2018. Architectural design of a Safe Mission Manager for Unmanned Aircraft Systems. FOURNAL OF SYSTEMS ARCHITECTURE 90, SI (OCT 2018), 94-108. https://doi.org/10.1016/j.sysarc.2018.09.003

[111] George Vachtsevanos, Benjamin Lee, Sehwan Oh, and Michael Balchanos. 2018. Resilient Design and Operation of Cyber Physical Systems with Emphasis on Unmanned Autonomous Systems. FOURNAL OF INTELLIGENT \& ROBOTIC SYSTEMS 91, 1, SI (JUL 2018), 59-83. https: //doi.org/10.1007/s10846-018-0881-x

[112] Bertold Van den Bergh and Sofie Pollin. 2019. Keeping UAVs Under Control During GPS Jamming. IEEE SYSTEMS JOURNAL 13, 2 (JUN 2019), 2010-2021. https://doi.org/10.1109/JSYST.2018.2882769

[113] Michael Vierhauser, Sean Bayley, Jane Wyngaard, Jinghui Cheng, Wandi Xiong, Robyn Lutz, Joshua Huseman, and Jane Cleland-Huang. 2018. Interlocking Safety Cases for Unmanned Autonomous Systems in Urban Environments. (2018), 416-417. https://doi.org/10.1145/3183440.3195035

[114] P. Volf. 2017. Simulation of UAS integration into shared airspace for validation of impact on ATM systems. In 2017 Integrated Communications, Navigation and Surveillance Conference (ICNS). 1-16.

[115] Xiao Wang, Jianfu Zhang, Pingfa Feng, Dingwen Yu, and Zhijun Wu. 2018. A Safety Monitoring System for Unmanned Aerial Vehicles. , Article 25 (2018), 8 pages. https://doi.org/10.1145/3207677.3278103

[116] Li Wei, Wang Ji Dong, and Li Meng Yang. 2017. A Vision-Based Attitude/Position Estimation for the Automatic Landing of Unmanned Helicopter on Ship. (2017), 1-5. https://doi.org/10.1145/3150978.3150992

[117] Graham Wild, John Murray, and Glenn Baxter. 2016. Exploring civil drone accidents and incidents to help prevent potential air disasters. Aerospace $3,3(2016), 22$.

[118] Jianfa Wu, Honglun Wang, Na Li, and Zikang Su. 2020. Formation Obstacle Avoidance : A Fluid-Based Solution. IEEE SYSTEMS fOURNAL 14, 1 (MAR 2020), 1479-1490. https://doi.org/10.1109/JSYST.2019.2917786

[119] Mei-Min Wu, Yu-Xiang Xiao, and Qian Bi. 2020. Software design of monitoring and flight simulation for UAV swarms based on OSGEarth. INTERNATIONAL JOURNAL OF COMPUTATIONAL SCIENCE AND ENGINEERING 21, 3 (2020), 346-354. https://doi.org/10.1504/IJCSE.2020.106059

[120] Chenchen Xu, Xiaohan Liao, Junming Tan, Huping Ye, and Haiying Lu. 2020. Recent Research Progress of Unmanned Aerial Vehicle Regulation Policies and Technologies in Urban Low Altitude. IEEE ACCESS 8 (2020), 74175-74194. https://doi.org/10.1109/ACCESS.2020.2987622

[121] Chao Yin, Zhenyu Xiao, Xianbin Cao, Xing Xi, Peng Yang, and Dapeng Wu. 2018. Offline and Online Search : UAV Multiobjective Path Planning Under Dynamic Urban Environment. IEEE INTERNET OF THINGS fOURNAL 5, 2, SI (APR 2018), 546-558. https://doi.org/10.1109/JIOT.2017.2717078

[122] Man-Ki Yoon, Bo Liu, Naira Hovakimyan, and Lui Sha. 2017. VirtualDrone : Virtual Sensing, Actuation, and Communication for Attack-Resilient Unmanned Aerial Systems. (2017), 143-154. https://doi.org/10.1145/3055004.3055010 
[123] Zhaoyue Zhang, Jing Zhang, Peng Wang, and Lei Chen. 2018. Research on Operation of UAVs in Non-isolated Airspace. CMC-COMPUTERS MATERIALS \& CONTINUA 57, 1 (OCT 2018), 151-166. https://doi.org/10.32604/cmc.2018.02890

[124] Yueyan Zhi, Zhangjie Fu, Xingming Sun, and Jingnan Yu. 2020. Security and Privacy Issues of UAV : A Survey. MOBILE NETWORKS \& APPLICATIONS 25, 1, SI (FEB 2020), 95-101. https://doi.org/10.1007/s11036-018-1193-x

[125] Wenyu Zhu and Richard Chapman. 2019. Stereo-Vision-Based Collision Avoidance Simulation. (2019), 156-159. https://doi.org/10.1145/3299815. 3314446 


\section{A APPENDICE : TABLEAU DE LA RSL SELON LA MÉTHODE QQOQCCP}

Déclinaison de la méthode QQOQCCP pour le soutien de la sécurité aux missions de drones et références - 1 sur 2

\begin{tabular}{|c|c|c|c|c|c|c|c|}
\hline Question & Question spécifique & Réponse & Nombre réponses & Références & $\begin{array}{l}\text { Pourcentage } \\
\text { réponses } \\
\text { sur total } \\
\text { articles }\end{array}$ & $\begin{array}{l}\text { Pourcentage } \\
\text { réponses } \\
\text { sur total } \\
\text { réponses }\end{array}$ & $\begin{array}{l}\text { Nombre } \\
\text { total de } \\
\text { réponses }\end{array}$ \\
\hline \multirow[t]{5}{*}{ Qui? } & \multirow{5}{*}{$\begin{array}{l}\text { les bénéficiaires des } \\
\text { contributions des } \\
\text { articles }\end{array}$} & régulateur & 6 & {$[6,11,53,69,92,120]$} & $6,06 \%$ & $4,76 \%$ & \multirow{5}{*}{126} \\
\hline & & $\begin{array}{l}\text { prestataire de ser- } \\
\text { vices de la naviga- } \\
\text { tion aérienne }\end{array}$ & 26 & $\begin{array}{l}{[3,4,9,12,16,24,25,36,38,39,47,54,59,60,64,67,68,} \\
76,88,95,102,113-115,120,123]\end{array}$ & $26,26 \%$ & $20,63 \%$ & \\
\hline & & fabricant & 12 & {$[6,27,37,58,69,73,82,89,92,109,122,124]$} & $12,12 \%$ & $9,52 \%$ & \\
\hline & & exploitant & 23 & $\begin{array}{l}{[3,4,10,11,16,21,25,33,36,38,39,59,64,66,74,81,} \\
88,93,95,102,113,115,120]\end{array}$ & $23,23 \%$ & $18,25 \%$ & \\
\hline & & télépilote & 59 & $\begin{array}{l}{[2,7,9,11,13-15,17,19,22,26-28,32,34,35,40,43,44,} \\
46,48,49,52,55-57,60,62,63,67,68,70-73,75,77,83- \\
86,90,91,94,96-98,103,104,106,107,110-112,116, \\
118,119,121,125]\end{array}$ & $59,60 \%$ & $46,83 \%$ & \\
\hline \multirow[t]{12}{*}{ Quoi? } & \multirow[t]{3}{*}{ la taille de UAS } & small & 64 & $\begin{array}{l}{[2,4,7,9-11,13,15-17,19,21,22,24-28,34,36,39,40,} \\
46,48,49,52,55,56,58-60,62,63,66-69,71,74-77,84- \\
86,88,91,93,94,97,98,103,104,106,107,112-116,120- \\
122,124]\end{array}$ & $64,65 \%$ & $62,14 \%$ & \multirow[t]{3}{*}{103} \\
\hline & & heavy & 23 & $\begin{array}{l}{[11,12,33-35,37,43,47,54,64,70,71,73,82,83,89,92,} \\
95,102,110,111,114,123]\end{array}$ & $23,23 \%$ & $22,33 \%$ & \\
\hline & & non mentionné & 16 & $\begin{array}{l}{[3,6,14,32,38,44,53,57,72,81,90,96,109,118,119,} \\
125]\end{array}$ & $16,16 \%$ & $15,53 \%$ & \\
\hline & \multirow[t]{3}{*}{$\begin{array}{l}\text { les aéronefs } \\
\text { autonomes sont-ils } \\
\text { inclus? }\end{array}$} & oui & 39 & $\begin{array}{l}{[4,6,12,14,25,27,28,32-35,43,44,48,49,52-54,56,62-} \\
64,73,74,76,86,91,92,97,98,102,110,111,113,114, \\
116,122,123,125]\end{array}$ & $39,39 \%$ & & \multirow[t]{3}{*}{99} \\
\hline & & non & 58 & $\begin{array}{l}{[2,7,9-11,13,15-17,19,21,22,24,26,36-40,46,47,55,} \\
57-60,66-72,75,77,81-85,88,89,93-96,103,104,106, \\
107,109,112,115,118-121,124]\end{array}$ & $58,59 \%$ & & \\
\hline & & non mentionné & 2 & {$[3,90]$} & $2,02 \%$ & & \\
\hline & \multirow[t]{3}{*}{$\begin{array}{l}\text { le genre d'UAS } \\
\text { considéré }\end{array}$} & Voilure fixe & 25 & $\begin{array}{l}{[11,12,33-37,43,47,54,64,66,70,71,73,75,82,83,88,} \\
95,102,110,111,115,123]\end{array}$ & $25,25 \%$ & $23,81 \%$ & \multirow[t]{3}{*}{105} \\
\hline & & Voilure tournante & 53 & $\begin{array}{l}{[2,7,9-11,13,15,17,21,22,25-28,40,46,48,49,52,55,} \\
56,58-60,62,63,67-69,71,74,75,77,84-86,88,89,93, \\
94,97,98,103,106,111-113,115,116,120-122,124]\end{array}$ & $53,54 \%$ & $50,48 \%$ & \\
\hline & & non mentionné & 27 & $\begin{array}{l}{[3,4,6,14,16,19,24,32,38,39,44,53,57,72,76,81,90-} \\
92,96,104,107,109,114,118,119,125]\end{array}$ & $27,27 \%$ & $25,71 \%$ & \\
\hline & \multirow[t]{3}{*}{ le segment du système } & air & 57 & $\begin{array}{l}{[6,7,10,11,13,14,17,22,26,27,32-35,40,43,44,46,48,} \\
49,52-54,56,57,59,60,62,63,70,73-75,77,82,84,86, \\
89-92,96,98,102,104,109-111,113,114,116,118,120- \\
122,124,125]\end{array}$ & $57,58 \%$ & $51,35 \%$ & \multirow[t]{3}{*}{111} \\
\hline & & communications & 12 & {$[6,11,21,33,53,58,93,103,107,112,113,124]$} & $12,12 \%$ & $10,81 \%$ & \\
\hline & & sol & 42 & $\begin{array}{l}{[2-4,6,9,11,12,15,16,19,24,25,28,33,36-39,47,53,} \\
55,64,66-69,71,72,76,81,83,85,88,94,95,97,106, \\
113,115,119,123,124]\end{array}$ & $42,42 \%$ & $37,84 \%$ & \\
\hline
\end{tabular}


Déclinaison de la méthode QQOQCCP pour le soutien de la sécurité aux missions de drones et références - 2 sur 2

\begin{tabular}{|c|c|c|c|c|c|c|c|}
\hline Question & Question spécifique & Réponse & Nombre réponses & Références & $\begin{array}{l}\text { Pourcentage } \\
\text { réponses } \\
\text { sur total } \\
\text { articles }\end{array}$ & $\begin{array}{l}\text { Pourcentage } \\
\text { réponses } \\
\text { sur total } \\
\text { réponses }\end{array}$ & $\begin{array}{l}\text { Nombre } \\
\text { total de } \\
\text { réponses }\end{array}$ \\
\hline \multirow[t]{16}{*}{ Où? } & \multirow{4}{*}{$\begin{array}{l}\text { caractéristiques de } \\
\text { l'espace aérien où } \\
\text { se passe la mission }\end{array}$} & non contrôlé & 2 & {$[35,73]$} & $2,02 \%$ & $1,96 \%$ & 102 \\
\hline & & contrôlé & 18 & $\begin{array}{l}{[9,12,32,33,38,43,47,53,54,64,66,71,82,92,102,110,} \\
114,123]\end{array}$ & $18,18 \%$ & $17,65 \%$ & \\
\hline & & $\begin{array}{l}\text { très basse altitude } \\
\text { (VLL) }\end{array}$ & 59 & $\begin{array}{l}{[2,4,6,10,11,13,15-17,19,24-27,34,36,38-40,44,46,} \\
49,55,56,58-60,62,63,67-69,72,74-77,81,84,86,88, \\
93,97,98,103,104,107,109-115,118-121,124]\end{array}$ & $59,60 \%$ & $57,84 \%$ & \\
\hline & & non mentionné & 23 & $\begin{array}{l}{[2,4,11,16,24,26,36,62,69,71,76,81,88,104,109,112,} \\
113,115,120,121]\end{array}$ & $23,23 \%$ & $22,55 \%$ & \\
\hline & \multirow{3}{*}{$\begin{array}{l}\text { les environnements } \\
\text { urbains sont-ils } \\
\text { concernés? }\end{array}$} & oui & 20 & $\begin{array}{l}{[2,4,11,16,24,26,36,62,69,71,76,81,88,104,109,112,} \\
113,115,120,121]\end{array}$ & $20,20 \%$ & & 99 \\
\hline & & non & 58 & $\begin{array}{l}{[6,9,10,12,13,15,17,19,25,27,32-35,38-40,43,44,} \\
46,47,49,52-56,58-60,63,64,66-68,72-75,77,82,84- \\
86,92,93,97,98,102,103,107,110,111,114,118,119, \\
123,124]\end{array}$ & $58,59 \%$ & & \\
\hline & & non mentionné & 21 & $\begin{array}{l}{[3,7,14,21,22,28,37,48,57,70,83,89-91,94-96,106,} \\
116,122,125]\end{array}$ & $21,21 \%$ & & \\
\hline & \multirow[t]{3}{*}{ type d'opérations } & VLOS & 12 & {$[11,15,28,38,69,75,94,95,103,114,120,124]$} & $12,12 \%$ & $11,21 \%$ & 107 \\
\hline & & BVLOS & 77 & $\begin{array}{l}{[2,4,7,9-14,16,17,19,24-28,32-36,38-40,43,44,46-} \\
49,52-56,58-60,62,64,66-77,81-84,86,88,92,94,97, \\
98,102,104,106,110-116,120-123]\end{array}$ & $77,78 \%$ & $71,96 \%$ & \\
\hline & & non mentionné & 18 & $\begin{array}{l}{[3,6,21,22,37,57,63,85,89-91,93,96,107,109,118,} \\
119,125]\end{array}$ & $18,18 \%$ & $16,82 \%$ & \\
\hline & \multirow[t]{3}{*}{$\begin{array}{l}\text { est-ce applicable à } \\
\text { la longue } \\
\text { élongation? }\end{array}$} & oui & 81 & $\begin{array}{l}{[2,4,6,9,10,12,14,16,17,19,22,24-28,32-40,43,46-} \\
49,52-56,58,60,62-64,66-73,75-77,81,82,84-86,88, \\
89,91-95,97,102-104,107,109-116,120-124]\end{array}$ & $81,82 \%$ & & 99 \\
\hline & & non & 7 & {$[7,15,59,74,83,98,106]$} & $7,07 \%$ & & \\
\hline & & non mentionné & 11 & {$[3,11,13,21,44,57,90,96,118,119,125]$} & $11,11 \%$ & & \\
\hline & \multirow{3}{*}{$\begin{array}{l}\text { les drones sont-ils } \\
\text { intégrés au trafic } \\
\text { aérien? }\end{array}$} & oui & 23 & $\begin{array}{l}{[4,9,12,33,36,39,43,44,47,53,54,64,66-68,71,73,} \\
82,92,102,104,114,123]\end{array}$ & $23,23 \%$ & & 99 \\
\hline & & non & 70 & $\begin{array}{l}{[2,6,7,10,11,13-17,19,21,22,24-28,32,34,35,37,} \\
38,40,46,48,49,52,55,56,59,60,62,63,69,70,72,74- \\
77,81,83-86,88,89,91,93-95,97,98,103,106,107,109- \\
113,115,116,118-122,124]\end{array}$ & $70,71 \%$ & & \\
\hline & & non mentionné & 6 & {$[3,57,58,90,96,125]$} & $6,06 \%$ & & \\
\hline \multirow[t]{6}{*}{ Quand? } & \multirow{6}{*}{$\begin{array}{l}\text { étapes de la } \\
\text { mission }\end{array}$} & réglementation & 2 & {$[11,53]$} & $2,02 \%$ & $1,85 \%$ & 108 \\
\hline & & $\begin{array}{ll}\text { fabrication } & \text { du } \\
\text { drone } & \end{array}$ & 5 & {$[3,37,46,82,111]$} & $5,05 \%$ & $4,63 \%$ & \\
\hline & & $\begin{array}{l}\text { préparation de la } \\
\text { mission }\end{array}$ & 21 & $\begin{array}{l}{[3,4,15,16,25,33,38,40,55,60,62,66,70,81,85,88,} \\
95,97,113,115,120]\end{array}$ & $21,21 \%$ & $19,44 \%$ & \\
\hline & & $\begin{array}{l}\text { demande d'autori- } \\
\text { sation }\end{array}$ & 3 & {$[36,38,39]$} & $3,03 \%$ & $2,78 \%$ & \\
\hline & & $\begin{array}{l}\text { réalisation de la } \\
\text { mission }\end{array}$ & 76 & $\begin{array}{l}{[2,6,7,9,10,12-15,17,19,22,24,26-28,32-35,43,44,} \\
47-49,52,54,56-60,63,64,67-69,71-77,83,84,86,89- \\
94,96,98,102-104,106,107,109-116,118-125]\end{array}$ & $76,77 \%$ & $70,37 \%$ & \\
\hline & & $\begin{array}{l}\text { après réalisation de } \\
\text { la mission }\end{array}$ & 1 & [21] & $1,01 \%$ & $0,93 \%$ & \\
\hline \multirow[t]{3}{*}{ Combien? } & \multirow[t]{3}{*}{$\begin{array}{l}\text { nombre de drones } \\
\text { impliqués dans } \\
\text { l'opération }\end{array}$} & un seul & 55 & $\begin{array}{l}{[4,6,10,11,15-17,21,22,26-28,33,34,36-38,40,43,} \\
46,48,52,54,55,57,58,62,66-70,74,75,81,83-86,88- \\
90,96,98,103,104,106,110-112,116,121,122,124,125]\end{array}$ & $55,56 \%$ & & 99 \\
\hline & & en essaim & 13 & {$[2,7,13,49,63,71,91,93,95,97,107,118,119]$} & $13,13 \%$ & & \\
\hline & & plusieurs drones & 31 & $\begin{array}{l}{[3,9,12,14,19,24,25,32,35,39,44,47,53,56,59,60} \\
64,72,73,76,77,82,92,94,102,109,113-115,120,123]\end{array}$ & $31,31 \%$ & & \\
\hline
\end{tabular}

